\title{
Horn Clause Contraction Functions
}

James P. Delgrande

School of Computing Science,

Simon Fraser University,

Burnaby, B.C., V5A $1 S 6$

Canada

Renata Wassermann

Dept. of Computer Science

University of São Paulo

05508-090 São Paulo,

Brazil
JIM@CS.SFU.CA

RENATA@IME.USP.BR

\begin{abstract}
In classical, AGM-style belief change, it is assumed that the underlying logic contains classical propositional logic. This is clearly a limiting assumption, particularly in Artificial Intelligence. Consequently there has been recent interest in studying belief change in approaches where the full expressivity of classical propositional logic is not obtained. In this paper we investigate belief contraction in Horn knowledge bases. We point out that the obvious extension to the Horn case, involving Horn remainder sets as a starting point, is problematic. Not only do Horn remainder sets have undesirable properties, but also some desirable Horn contraction functions are not captured by this approach. For Horn belief set contraction, we develop an account in terms of a model-theoretic characterisation involving weak remainder sets. Maxichoice and partial meet Horn contraction is specified, and we show that the problems arising with earlier work are resolved by these approaches. As well, constructions of the specific operators and sets of postulates are provided, and representation results are obtained. We also examine Horn package contraction, or contraction by a set of formulas. Again, we give a construction and postulate set, linking them via a representation result. Last, we investigate the closely-related notion of forgetting in Horn clauses. This work is arguably interesting since Horn clauses have found widespread use in AI; as well, the results given here may potentially be extended to other areas which make use of Horn-like reasoning, such as logic programming, rule-based systems, and description logics. Finally, since Horn reasoning is weaker than classical reasoning, this work sheds light on the foundations of belief change.
\end{abstract}

\section{Introduction}

The area of belief change in knowledge representation studies how a rational agent may alter its beliefs in the presence of new information. The best-known approach in this area is the so-called AGM paradigm (Alchourrón, Gärdenfors, \& Makinson, 1985; Gärdenfors, 1988), named after the original developers. This work focused primarily on two belief change operations, belief contraction, in which an agent may reduce its stock of beliefs, and belief revision, in which new information is consistently incorporated into the agent's belief corpus. A fundamental assumption of this approach is that the underlying logic governing 
the agent's beliefs subsumes classical propositional logic. However, in artificial intelligence (AI) a major concern is with efficient, limited, and ideally tractable reasoning. Hence there has been significant effort in studying limited reasoners, including Horn clause based approaches, limited epistemic reasoning involving explicit belief (Lakemeyer \& Levesque, 2000), and description logics (Baader, Calvanese, McGuiness, Nardi, \& Patel-Schneider, 2007). Moreover, since a knowledge base will evolve, it is crucially important that change in a knowledge base be managed in a principled fashion. However, the AGM approach cannot be used as a guide to change in any approach, such as those mentioned above, that does not subsume classical propositional logic.

In this paper we address belief change in the expressively-weak language of Horn clauses, where a Horn clause can be written as a rule in the form $a_{1} \wedge a_{2} \wedge \cdots \wedge a_{n} \rightarrow a$ for $n \geq 0$, and where $a, a_{i}(1 \leq i \leq n)$ are atoms. (Thus, expressed in conjunctive normal form, a Horn clause will have at most one positive literal.) In our approach, an agent's beliefs are represented by a Horn clause knowledge base, and the input is a conjunction of Horn clauses. We focus on belief contraction (and, later, operators related to contraction) in which the agent's stock of beliefs decreases.

The topic of Horn clause contraction (and the general topic of Horn belief change in general) is interesting for several reasons. First, Horn clause reasoners constitute an important class of AI systems, and Horn clauses have found extensive use in artificial intelligence and database theory, in areas such as logic programming, truth maintenance systems, and deductive databases. Horn clause belief change also sheds light on the theoretical underpinnings of belief change, in that it weakens the assumption that the underlying logic contains propositional logic. Hence results obtained here may be relevant to belief change in other areas of limited reasoning. For example, approaches to explicit belief often derives much of their inspiration from relevance logic (Anderson \& Belnap Jr., 1975); and description logics, while constituting fragments of classical first-order logic, nonetheless in many cases do not support full propositional reasoning. ${ }^{1}$

Creignou, Papini, Pichler, and Woltran (2012) provide further motivation for the study of belief change in tractable fragments of propositional logic:

In many applications, the language is restricted a priori. For instance, a rulebased formalization of expert knowledge is much easier to handle for standard users. In case users want to revise some rules, they indeed expect that the outcome is still in the easy-to-read format they are used to. Many fragments of propositional logic allow for efficient reasoning methods. Suppose an agent who frequently has to answer queries about his beliefs. This should be done efficiently thus the beliefs are stored as a formula known to be in a tractable class. In case the beliefs of the agent are undergoing a revision, it is desired that the result of such an operation yields a formula in the same fragment. Hence, the agent still can use the dedicated solving method he is equipped with for this fragment. In case such changes are performed rarely, we do not bother whether the revision itself can be performed efficiently, but it is more important that the outcome can still be evaluated efficiently.

1. In fact, as Booth, Meyer, and Varzinczak (2009) point out, results here are also relevant to belief change in description logics, a topic that has also elicited recent interest. 
Horn clause contraction has become a topic of interest in belief change in recent years (Delgrande, 2008; Delgrande \& Wassermann, 2010, 2011; Booth et al., 2009; Booth, Meyer, Varzinczak, \& Wassermann, 2011; Zhuang \& Pagnucco, 2010a, 2011, 2012). As we discuss in the next section, most of this work centers on the notion of a remainder set, or a maximal subset of a knowledge base that fails to imply a given formula. We show that remainder sets in the Horn case are too restricted and cannot give all feasible contraction operators. As well they yield contraction operators with undesirable properties.

We propose the notion of a weak remainder set that serves as a basis for generating all Horn maxichoice contraction operators. Contraction is also considered in terms of the underlying model theory, a viewpoint that proves highly enlightening for studying Horn belief change. Given a specification for maxichoice contraction based on weak remainders, we go on to develop a specification for partial meet Horn contractions, and consider package contraction and forgetting. In all the contraction operators developed, we provide postulate sets along with constructions, and show representation results. Consequently we present a comprehensive exploration of the landscape of Horn contraction.

The next section introduces belief change while the following section discusses reasoning in Horn clause theories. The main approach is presented in Section 4, while Section 5 discusses considerations pertaining to the supplementary contraction postulates. Section 6 covers the related operators of package contraction and forgetting on Horn theories. The paper concludes with a discussion and a concluding section. Proofs are given in an appendix. Some of this material was presented previously by Delgrande (2008) and Delgrande and Wassermann (2010, 2011).

\section{Background}

In this section, we introduce the main concepts of the area of Belief Change which we will need throughout the paper.

\subsection{Belief Change}

As previously mentioned, the AGM approach (Alchourrón et al., 1985; Gärdenfors, 1988) is the original and best-known approach to belief change. ${ }^{2}$ The goal in this approach is to describe belief change at the knowledge level, that is, on an abstract level and independent of how beliefs are represented and manipulated. Belief states are modelled by sets of sentences, called belief sets, closed under the logical consequence operator of a logic that includes classical propositional logic in a language $\mathcal{L}$. Thus a belief set $K$ satisfies the constraint:

$$
\text { If } K \text { logically entails } \phi \text { then } \phi \in K \text {. }
$$

The central operators ${ }^{3}$ addressed are contraction, in which an agent reduces its set of beliefs, and revision, in which an agent consistently incorporates a new belief. In revision, since the new belief may be inconsistent with an agent's beliefs, some beliefs may need to be dropped in order to maintain a consistent set of beliefs. A third operator, belief expansion was also

2. As well, Peppas (2008) provides an excellent survey.

3. In this paper, we use the terms operator and function interchangeably when refering to belief change operations. 
introduced: For belief set $K$ and formula $\phi$, the expansion of $K$ by $\phi$, denoted $K+\phi$, is the deductive closure of $K \cup\{\phi\}$. Expansion captures the simplest form of belief change; it can be reasonably applied when new information is consistent with a belief set

These operators are characterised by two means. On the one hand, a set of rationality postulates for a belief change function may be provided; these postulates stipulate constraints that should govern any rational belief change function. On the other hand, specific constructions for a belief change function are given. Representation results are then provided, showing that a set of rationality postulates exactly captures the operator given by a particular construction.

We review these notions for belief contraction. Informally, the contraction of a belief set by a formula is a belief set in which that formula is not believed. Formally, a contraction function - is a function from $2^{\mathcal{L}} \times \mathcal{L}$ to $2^{\mathcal{L}}$ satisfying the following postulates.

$(K \dot{-} 1) \quad K \dot{-} \phi$ is a belief set.

$(K \dot{-} 2) \quad K \dot{-} \phi \subseteq K$.

$(K \dot{-} 3)$ If $\phi \notin K$, then $K \dot{-} \phi=K$.

$(K \dot{-} 4)$ If $\forall \phi$, then $\phi \notin K \dot{-} \phi$.

$(K \dot{-} 5)$ If $\phi \in K$, then $K \subseteq(K \dot{-} \phi)+\phi$.

$(K \dot{-} 6)$ If $\phi \equiv \psi$, then $K \dot{-} \phi=K \dot{-} \psi$.

$(K \dot{-} 7) K \dot{-} \phi \cap K \dot{-} \psi \subseteq K \dot{-}-(\phi \wedge \psi)$.

$(K \dot{-} 8)$ If $\psi \notin K \dot{-}(\phi \wedge \psi)$ then $K \dot{-}(\phi \wedge \psi) \subseteq K \dot{-} \psi$.

Thus, contraction yields a belief set $(K \dot{-} 1)$ in which the sentence for contraction $\phi$ is not believed (unless $\phi$ is a tautology) $(K \dot{-} 4)$. No new sentences are believed $(K \dot{-} 2)$, and if the formula is not originally believed then contraction has no effect $(K \dot{-} 3)$. The fifth postulate, the so-called recovery postulate, states that nothing is lost if one contracts and expands by the same sentence. This postulate is controversial, as discussed, for example by Hansson (1999). The sixth postulate asserts that contraction is independent of how a sentence is syntactically expressed. The last two postulates express relations between contracting by conjunctions and contracting by the constituent conjuncts. Hence $(K \dot{-} 7)$ says that if a formula is in the result of contracting by each of two formulas then it is in the result of contracting by their conjunction. $(K \dot{-} 8)$ says that if a conjunct is not in the result of contracting by a conjunction, then, in the presence of $(K \dot{-} 7)$, contracting by that conjunct is the same as contracting by the conjunction. The first six postulates are referred to as the basic postulates while the last two are referred to as the supplementary postulates.

Revision represents the situation in which new information may be inconsistent with the reasoner's beliefs $K$, and needs to be incorporated in a consistent manner, the one exception being when the formula for revision itself is inconsistent. A revision function $*$ is a function from $2^{\mathcal{L}} \times \mathcal{L}$ to $2^{\mathcal{L}}$ satisfying a set of postulates analogous to those for contraction. Contraction is usually taken as being the more fundamental operator for belief 
change. Moreover, revision and contraction are interdefinable. Revision can be defined in terms of contraction by means of the Levi Identity:

$$
K * \phi=(K \dot{-} \neg \phi)+\phi .
$$

Thus, to revise by $\phi$, make $K$ consistent with $\phi$ then expand by $\phi$. Contraction can be similarly defined in terms of revision by the Harper identity:

$$
K \dot{-} \phi=K \cap(K * \neg \phi) .
$$

Since we do not consider revision functions in this paper, we refer the reader to to the work of Gärdenfors (1988) and Peppas (2008) for details.

Various constructions have been proposed to characterise belief change. The original construction was in terms of remainder sets, where a $\phi$-remainder of $K$ is a maximal subset of $K$ that fails to imply $\phi$. Formally:

Definition 1 Let $K \subseteq \mathcal{L}$ and let $\phi \in \mathcal{L}$.

$K \downarrow \phi$ is the set of sets of formulas s.t. $K^{\prime} \in K \downarrow \phi$ iff

1. $K^{\prime} \subseteq K$

2. $K^{\prime} \nvdash \phi$

3. For any $K^{\prime \prime}$ s.t. $K^{\prime} \subset K^{\prime \prime} \subseteq K$, it holds that $K^{\prime \prime} \vdash \phi$.

Each $K^{\prime} \in K \downarrow \phi$ is a $\phi$-remainder of $K$.

Thus $K \downarrow \phi$ is the class of all maximal $\phi$-nonimplying subsets of $K$. When there is no ambiguity, we will also refer to $K^{\prime} \in K \downarrow \phi$ as simply a remainder of $K$.

Two classes of contraction functions are relevant for our concerns. In maxichoice contraction, contraction is defined to correspond to a single selected remainder. In partial meet contraction, contraction corresponds to the intersection of some subset of the remainders. Consequently, any maxichoice contraction is a partial meet contraction but not vice versa.

From a logical point of view, the $\phi$-remainders comprise equally-good candidates for a contraction of $\phi$ from $K$. Selection functions are introduced to reflect the extra-logical factors that need to be taken into account, to obtain the "best" or most plausible remainders. In maxichoice contraction, the selection function determines a single selected remainder as the contraction. In partial meet contraction, the selection function returns a subset of the remainders, the intersection of which constitutes the contraction. Thus if the selection function is denoted by $\gamma(\cdot)$, then the contraction of $K$ by formula $\phi$ can be expressed by

$$
K \dot{-} \phi=\bigcap \gamma(K \downarrow \phi) \text {. }
$$

For belief set $K$ and function - from $2^{\mathcal{L}} \times \mathcal{L}$ to $2^{\mathcal{L}}$, it proves to be the case that $\dot{-}$ is a partial meet contraction function iff it satisfies the basic contraction postulates $(K \dot{-}-1)$ $(K \dot{-} 6)$. Last, let $\preceq$ be a transitive relation on $2^{K}$, and let the selection function be defined by:

$$
\gamma(K \downarrow \phi)=\left\{K^{\prime} \in K \downarrow \phi \mid \forall K^{\prime \prime} \in K \downarrow \phi, K^{\prime \prime} \preceq K^{\prime}\right\} .
$$

$\gamma$ is a transitively relational selection function, and $\dot{-}$ defined in terms of such a $\gamma$ is a transitively relational partial meet contraction function. Then we have: 
Theorem 1 (Alchourrón et al., 1985) Let $K$ be a belief set and let $\dot{-}$ be a function from $2^{\mathcal{L}} \times \mathcal{L}$ to $2^{\mathcal{L}}$. Then

1. - is a partial meet contraction function iff it satisfies the contraction postulates $(K \dot{-} 1)-(K \dot{-} 6)$.

2. - is a transitively relational partial meet contraction function iff it satisfies the contraction postulates $(K \dot{-}-1)-(K \dot{-} 8)$.

The second major construction for contraction functions is called epistemic entrenchment. The general idea is that extra-logic factors related to contraction are given by an ordering on formulas in the agent's belief set, reflecting how willing the agent would be to give up a formula. Then a contraction function can be defined in terms of removing less entrenched formulas from the belief set. Gärdenfors and Makinson (1988) show that for logics including classical propositional logic, the two types of constructions, selection functions over remainder sets and epistemic entrenchment orderings, capture the same class of contraction functions.

Two other constructions were also proposed in the literature and shown to be equivalent to transitively relational partial meet contraction: safe contraction (Alchourron \& Makinson, 1985; Rott, 1992) and systems of spheres (Grove, 1988). We do not address either construction in this paper.

\subsection{Belief Change and Horn Clause Theories}

Earlier work on belief change and Horn theories focussed on specific aspects of the problem, rather than on a general characterisation of Horn clause belief change. For example, the complexity of specific approaches to revising knowledge bases has been addressed by Eiter and Gottlob (1992). This includes the case where the knowledge base and formula for revision are conjunctions of Horn clauses, although the results of revision may not be Horn. Not unexpectedly, results are generally better in the Horn case. Liberatore (2000) considers the problem of a compact representation for revision in the Horn case. Given a knowledge base $K$ and formula $\phi$, both Horn, the main problem addressed is whether the knowledge base, revised according to a given operator, can be expressed by a propositional formula whose size is polynomial with respect to the sizes of $K$ and $\phi$.

Langlois, Sloan, Szörényi, and Turán (2008) approach the study of revising Horn formulas by characterising the existence of a complement of a Horn consequence; such a complement corresponds to the result of a contraction operator. This work may be seen as a specific instance of a general framework developed by Flouris, Plexousakis and Antoniou (2004). They study belief change under a broad notion of logic. In particular, they give a criterion for the existence of a contraction operator satisfying the basic AGM postulates in terms of decomposability.

The present paper builds on and extends (Delgrande, 2008; Delgrande \& Wassermann, 2010, 2011). Delgrande (2008) addresses maxichoice belief contraction in Horn clause theories, where contraction is defined in terms of remainder sets, using Definition 1, but expressed in terms of derivations among Horn clauses. Booth, Meyer, and Varzinczak (2009) and then Booth, Meyer, Varzinczak, and Wassermann (2011) further develop this area, by 
considering other versions of contraction, all based on remainder sets: partial meet contraction, a generalisation of partial meet, and package contraction. Horn contraction based on remainders was found to be inadequate by Delgrande and Wassermann (2010), and instead they developed a notion of weak remainder. The work by Zhuang and Pagnucco (2010a, 2012) follows another line, focusing on epistemic entrenchment and model-based constructions. These approaches are discussed and compared in more detail once we have introduced our overall approach.

Recently, revision operations for Horn theories have also been developed (Delgrande \& Peppas, 2011), and revision in other fragments of propositional logic has also been explored (Creignou et al., 2012). However the relation of this work with the contraction operations described in this paper is still unclear.

\section{Horn Clause Theories}

We will deal with languages based on finite sets of atoms, or propositional letters $\mathbf{P}=$ $\{a, b, c, \ldots\}$, where $\mathbf{P}$ includes the distinguished atom $\perp . \mathcal{L}$ is the language of propositional logic over $\mathbf{P}$ and with the usual connectives $\neg, \wedge, \vee$, and $\rightarrow .{ }^{4} \mathcal{L}_{H C}$ is the restriction of $\mathcal{L}$ to Horn formulas, where a Horn formula is a finite conjunction of Horn clauses. $\mathcal{L}_{H C}$ is the least set given by:

1. $a_{1} \wedge a_{2} \wedge \cdots \wedge a_{n} \rightarrow a$, where $n \geq 0$, and $a, a_{i}(1 \leq i \leq n)$ are atoms, is a Horn clause.

2. Every Horn clause is a Horn formula.

3. If $\phi$ and $\psi$ are Horn formulas then so is $\phi \wedge \psi$.

As well, for convenience, $\top$ will be taken as denoting $a \rightarrow a$ for some specific atom $a$. For a Horn clause $r$ as in 1 above, if $n=0$ then $r$ is a fact, and $\rightarrow a$ is also written as $a$. For a Horn clause $r$ as in 1 above, head $(r)$ is $a$, and $\operatorname{body}(r)$ is the set $\left\{a_{1}, a_{2}, \ldots, a_{n}\right\}$. If $a$ is a fact, then $\operatorname{head}(r)$ is $a$, and $\operatorname{body}(r)$ is empty. If for a Horn clause $r$ we have that $h e a d(r)=\perp$, then $r$ is an integrity constraint. Allowing conjunctions of clauses, as given in 3 , adds nothing of interest to the expressibility of the language with respect to reasoning. However, it adds to the expressibility of contraction, as we are able to contract by more than a single Horn clause.

Semantics: An interpretation of $\mathcal{L}$ is a function from $\mathbf{P}$ to $\{$ true, false $\}$ such that $\perp$ is assigned false. Sentences of $\mathcal{L}$ are true or false in an interpretation according to the standard rules of propositional logic. An interpretation $M$ is a model of a sentence $\phi$ (or set of sentences), written $M=\phi$, just if $\phi$ is true in $M \cdot \operatorname{Mod}(\phi)$ is the set of models of formula (or set of formulas) $\phi$; thus $\operatorname{Mod}(\top)$ is the set of interpretations of $\mathcal{L}$. An interpretation is usually identified with the atoms true in that interpretation. Thus, for the language given by $\mathbf{P}=\{p, q, r, s\}$, the interpretation expressed by $\{p, q\}$ is that in which $p$ and $q$ are true and $r$ and $s$ are false. For convenience, we also will express interpretations by juxtaposition of atoms. Thus the set of interpretations $\{\{p, q\},\{p\},\{\}\}$ will usually be written as $\{p q, p, \emptyset\}$.

All of these notions are inherited by the corresponding Horn formula language $\mathcal{L}_{H C}$. A key point concerning Horn theories is that such theories are characterised semantically by

4. To avoid clutter, and because no ambiguity results, we don't parameterize $\mathcal{L}$ by $\mathbf{P}$. 
the fact that their models are closed under intersections of positive atoms in an interpretation. That is, a Horn theory $H$ satisfies the constraint:

$$
\text { If } M_{1}, M_{2} \in \operatorname{Mod}(H) \text { then } M_{1} \cap M_{2} \in \operatorname{Mod}(H) \text {. }
$$

This leads to the notion of the characteristic models (Khardon, 1995) of a Horn formula or set of formulas: $M$ is a characteristic model of formula $\phi$ just if for every $M_{1}, M_{2} \in \operatorname{Mod}(\phi)$, $M_{1} \cap M_{2}=M$ implies that $M=M_{1}$ or $M=M_{2}$. Thus for example, $\{p \wedge q \rightarrow \perp, r\}$ has models $\{p r, q r, r\}$ and characteristic models $\{p r, q r\}$. Since $p r \cap q r=r, r$ isn't a characteristic model of $\phi$.

Proof Theory: We assume a suitable inference relation $\vdash$ for classical propositional logic. The following axioms and rules give an inference relation for Horn formulas, where for simplicity, $a$ and $b$, possibly subscripted, are taken as ranging over atoms.

Axioms: $\quad \perp \rightarrow a \quad a \rightarrow a$

\section{Rules:}

1. From $a_{1} \wedge \cdots \wedge a_{n} \rightarrow a$ and $b_{1} \wedge \cdots \wedge b_{n} \rightarrow a_{1}$

infer $b_{1} \wedge \cdots \wedge b_{n} \wedge a_{2} \wedge \cdots \wedge a_{n} \rightarrow a$

2. From $a_{1} \wedge \cdots \wedge a_{n} \rightarrow a$ infer $\quad a_{1} \wedge \cdots \wedge a_{n} \wedge b \rightarrow a$

3. For Horn clauses $r_{1}, r_{2}$, if $\operatorname{body}\left(r_{1}\right)=\operatorname{body}\left(r_{2}\right)$ and $\operatorname{head}\left(r_{1}\right)=\operatorname{head}\left(r_{2}\right)$ then from $r_{1}$ infer $r_{2}$.

4. (a) From $\phi \wedge \psi$ infer $\phi$ and $\psi$

(b) From $\phi$ and $\psi$ infer $\phi \wedge \psi$

Rule 1 is an extended version of modus ponens, while Rule 2 is strengthening of the antecedent. Rule 3 states that the order of atoms in the body of a Horn clause is irrelevant, as are repeated atoms.

A formula $\psi$ can be derived from a set of formulas $A$, written $A \vdash_{H C} \psi$, just if $\psi$ can be obtained from $A$ by a finite number of applications of the above rules and axioms; for simplicity we drop the subscript and write $A \vdash \psi$. If $A=\{\phi\}$ is a singleton set then we just write $\phi \vdash \psi$. A set of formulas $A \subseteq \mathcal{L}_{H C}$ is inconsistent just if $A \vdash \perp$. We use $\phi \equiv \psi$ to represent logical equivalence, that is $\phi \vdash \psi$ and $\psi \vdash \phi$.

Notation: We collect here for reference notation that is used in the paper. Lower-case Greek characters $\phi, \psi, \ldots$, possibly subscripted, denote arbitrary formulas of either $\mathcal{L}$ or $\mathcal{L}_{H C}$. Upper case Roman characters $A, B, \ldots$, possibly subscripted, denote arbitrary sets of formulas. $H, H_{1}, H^{\prime}$, etc. denote Horn belief sets, so that $\phi \in H$ iff $H \vdash_{H C} \phi$.

$C n(A)$ is the (classical, propositional) deductive closure of $A$ where $A$ is a formula or set of formulas of propositional logic. $C n^{h}(A)$ is the deductive closure of a Horn formula or set of formulas $A$ under Horn derivability. For set of formulas $A, \operatorname{Horn}(A)=\{\phi \in A \mid$ $\phi$ is a Horn formula\}.

We use $m$ (possibly subscripted) to denote a maximal consistent Horn theory; that is, $m \forall \perp$ and for every Horn formula $\phi$, either $\phi \in m$ or $m \cup\{\phi\} \vdash \perp$. Hence such a 
$m$ has exactly one model. We often use maximal consistent sets of formulas in place of interpretations, as it makes the statement and proof of various results easier. $|\phi|$ is the set of maximal, consistent Horn theories that contain $\phi$.

$M\left(M_{1}, M^{\prime}\right.$, etc.) will denote (classical, propositional) interpretations over some understood language. $\operatorname{Mod}(A)$ is the set of models of $A$. Arbitrary sets of interpretations will be denoted $\mathcal{M}\left(\mathcal{M}^{\prime}\right.$ etc. $)$. $C l_{\cap}(\mathcal{M})$ is the intersection closure of a set of interpretations $\mathcal{M}$; that is, $C l_{\cap}(\mathcal{M})$ is the least set of interpretations such that

1. $\mathcal{M} \subseteq C l_{\cap}(\mathcal{M})$ and

2. $M_{1}, M_{2} \in C l_{\cap}(\mathcal{M})$ implies that $M_{1} \cap M_{2} \in C l_{\cap}(\mathcal{M})$.

Note that $M$ denotes an interpretation expressed as a set of atoms, while $m$ denotes a maximal consistent set of Horn formulas. Thus the logical content is the same, in that an interpretation defines a maximal consistent set of Horn formulas, and vice versa. We retain these two interdefinable notations, since each is useful in the subsequent development. Similar comments apply to $\operatorname{Mod}(\phi)$ vs. $|\phi|$; we also make use of the fact that there is a 1-1 correspondence between elements of $|\phi|$ and of $\operatorname{Mod}(\phi)$.

Last, since $\mathbf{P}$ is finite, a (Horn or propositional logic) belief set may be finitely represented, that is, for $X$ a belief set, there is a formula $\phi$ such that $C n(\phi)=X$.

\section{Horn Clause Belief Set Contraction}

In this section, we examine the possible constructions for the operation of contraction of Horn belief sets. We begin by operations based on remainde sets and proceed to introducing the concept of a weak remainder set.

\subsection{Horn Clause Contraction and Remainder Sets}

The most straightforward way to define a Horn contraction function is by adapting a construction used in classical logic for contraction. To this end, Delgrande (2008) developed a remainder-set approach to Horn contraction, which was subsequently generalised by Booth, Meyer and Varzinczak (2009). It proves to be the case that these approaches are not sufficiently expressive for general Horn contraction; as well, contraction based on remainder sets can be shown to have undesirable properties. We review the pertinent aspects of these approaches here, and in particular consider why the results of (classical, AGM) contraction do not readily extend to the Horn case.

The definition of remainder sets for Horn clause belief sets (called e-remainder sets in Delgrande, 2008) is the same as that for a remainder set (Definition 1) but with respect to Horn clauses and Horn derivability. For $H$ a Horn belief set and $\phi \in \mathcal{L}_{H C}$, the set of e-remainders with respect to $H$ and $\phi$ is denoted by $H \downarrow_{e} \phi$.

Definition 2 Let $H \subseteq \mathcal{L}_{H C}$ and let $\phi \in \mathcal{L}_{H C}$.

$H \downarrow_{e} \phi$ is the set of sets of formulas such that $H^{\prime} \in H \downarrow_{e} \phi$ iff

1. $H^{\prime} \subseteq H$

2. $H^{\prime} \nvdash \phi$ 
3. For any $H^{\prime \prime}$ such that $H^{\prime} \subset H^{\prime \prime} \subseteq H$ it holds that $H^{\prime \prime} \vdash \phi$.

Each $H^{\prime} \in H \downarrow_{e} \phi$ is an $\phi$-e-remainder with respect to $H$.

Usually such a $H^{\prime}$ will just be referred to simply as a remainder, since the Horn context and underlying formula are clear.

Observation 1 If $H \downarrow_{e} \phi_{1}=H \downarrow_{e} \phi_{2}$, then for any $H^{\prime} \subseteq H, \phi_{1} \in C n^{h}\left(H^{\prime}\right)$ iff $\phi_{2} \in$ $C n^{h}\left(H^{\prime}\right)$.

Observation 2 (Upper bound property) If $X \subseteq H$ and $\phi \notin C n^{h}(X)$, then there is some $X^{\prime}$ such that $X \subseteq X^{\prime} \in H \downarrow$ e $\phi$.

Horn remainders as given in Definition 2 can be regarded as comprising a set of candidate contractions for $H$ by a formula $\phi$; a single such remainder then could be selected as the maxichoice contraction of $H$ by $\phi$. Booth, Meyer, and Varzinczak (2009) subsequently argue that maxichoice contraction is not sufficient for an account of Horn contraction functions. In classical AGM contraction, the set of partial meet contraction functions is defined by taking the intersection of some of the remainders. However, Booth, Meyer, and Varzinczak also argue that the set of Horn partial meet contractions is not sufficient to capture the full range of possible contraction functions. Instead they define infra remainder sets, as follows:

Definition 3 For belief sets $H$ and $X, X \in H \Downarrow_{e} \phi^{5}$ iff there is some $X^{\prime} \in H \downarrow_{e} \phi$ such that

$$
\left(\bigcap \downarrow_{\downarrow} \phi\right) \subseteq X \subseteq X^{\prime}
$$

The elements of $H \Downarrow_{e} \phi$ are the infra e-remainder sets of $H$ with respect to $\phi$.

Thus an infra e-remainder set is any belief set that contains the intersection of Horn remainders, and is contained in some Horn remainder. All e-remainder sets are clearly infra e-remainder sets, as is the intersection of any set of e-remainder sets. That is:

Observation 3 Let $H \subseteq \mathcal{L}_{H C}, \phi \in \mathcal{L}_{H C}$, and let $X \subseteq H \downarrow_{e} \phi$. Then $(\bigcap X) \in H \Downarrow_{e} \phi$.

Example 1 For $\mathbf{P}=\{a, b, c\}$, let $H=C n^{h}(a \wedge b)$.

Consider candidates for $H-(a \wedge b)$.

It can be verified that there are three remainder sets:

$$
\begin{aligned}
& \mathcal{C} n^{h}(a \wedge(c \rightarrow b)), \\
& \mathcal{C} n^{h}(b \wedge(c \rightarrow a)), \quad \text { and } \\
& \mathcal{C} n^{h}((a \rightarrow b) \wedge(b \rightarrow a) \wedge(c \rightarrow a) \wedge(c \rightarrow b)) .
\end{aligned}
$$

As well, any remainder set and any infra remainder set must contain the closure of $(c \rightarrow a) \wedge(c \rightarrow b)$.

5. Booth, Meyer, and Varzinczak (2009) write $X \in H \Downarrow_{e} \Phi$ where $\Phi$ is a set of Horn clauses. 
To see the last part of the example, note that both $(c \rightarrow a)$ and $(c \rightarrow b)$ are in all remainders, and so in the intersection of the remainders. This however leads to a significant blemish. Call $p$ inessential in $H$ if for any conjunction of atoms body not containing $p, H \vdash p \wedge$ body $\rightarrow a$ implies that either $\vdash p \wedge$ body $\rightarrow a$ or $H \vdash \operatorname{body} \rightarrow a$. For contraction defined in terms of remainder sets, or intersections of remainder sets, or infra remainder sets, we have the result: 6

Theorem 2 Let $\dot{-}$ be a Horn contraction function defined via a selection function as in (2) and based on (infra) remainder sets.

For $\phi \in H$ and $p$ inessential in $H$, we obtain that $(H \dot{-} \phi)+p \vdash \phi$.

The following example (based on an example in Hansson, 1999) illustrates the problem:

1. You believe Cleopatra had a son and a daughter $(s \wedge d)$.

2. You learn that the source of information was unreliable, so you remove this belief; i.e. you compute the contraction $H \dot{-}(s \wedge d)$.

3. You learn that it is raining outside $(r)$.

4. You conclude that Cleopatra had a son and daughter $(s \wedge d)$

This behaviour is clearly undesirable. However, consider what this example implies about Horn contraction to this point: We have that $H \dot{-}(s \wedge d)+r$ entails $s \wedge d$. Hence, regardless of how $\dot{-}$ is defined in terms of (infra) remainders, all models of $H \dot{-}(s \wedge d)$ in which $r$ is true must have that $s$ and $d$ is also true. What this in turn means is that $s \bar{d} r$ cannot be a model of any $s \wedge d$-remainder. This last point is curious, in that $s \bar{d} r$ is clearly a counter-model of $s \wedge d$, yet it does not take part in any remainder, and so does not take part in any contraction.

In AGM contraction, we have that each $\phi$-remainder of a belief set $K$ can be characterized by the set of models of $K$ together with a single countermodel of $\phi$, and vice versa (e.g., see Gärdenfors, 1988, p. 86). What the above example shows is that this equivalence between the proof-theoretic notion of remainders and the semantic notion of minimallyextended sets of models breaks down in the Horn case.

So, consider what is going on in the Horn case: Assume that $H \models \phi$ and we wish to find a maximal belief set $H^{\prime}$ such that $H^{\prime} \subset H$ and $H^{\prime} \not \models \phi$. That is, $H^{\prime}$ is to be a $\phi$-remainder set of $H$. As described, in classical AGM (maxichoice) contraction, from the semantic side one adds a countermodel of $\phi$ to the models of $H$; this set of models characterises a candidate theory for maxichoice contraction.

Consider the analogous process for Horn theories. Since a remainder set must be a Horn theory, and the models of a Horn theory are closed under intersection, we would need to make sure that this constraint holds here. So, intuitively, to carry out maxichoice Horn contraction, we would add a countermodel of the formula for contraction, and close the result under intersection. However, the theories resulting from this approach do not correspond to those obtained via remainder sets. To see this, consider again Example 1, and where the pertinent results are summarised in Figure 1.

6. This result would also be obtained in package contraction, discussed in Section 6, if package contraction were defined in terms of infra remainder sets. 


\begin{tabular}{|c|c|c|c|}
\hline $\begin{array}{c}\text { counter- } \\
\text { model }\end{array}$ & $\begin{array}{c}\text { induced } \\
\text { models }\end{array}$ & resulting KB & $\begin{array}{c}\text { remainder } \\
\text { set? }\end{array}$ \\
\hline \hline$a c$ & $a$ & $a \wedge(c \rightarrow b)$ & \\
\hline$a$ & & $b$ & $\sqrt{ }$ \\
\hline$b c$ & $b$ & $b \wedge(c \rightarrow a)$ & $\sqrt{ }$ \\
\hline$b$ & & $(a \rightarrow b) \wedge(b \rightarrow a)$ & \\
\hline$c$ & $\emptyset$ & $(a \rightarrow b) \wedge(b \rightarrow a) \wedge(c \rightarrow a) \wedge(c \rightarrow b)$ & $\sqrt{ }$ \\
\hline$\emptyset$ & &
\end{tabular}

Figure 1: Example: Candidates for Horn contraction

We have that $a c$ (viz. $a \bar{b} c$ ) is a countermodel of $\phi=a b$; this is given in the first entry of the first row of the table. Since $H$ has a model $a b$, the intersection of these models, $a b \cap a c=a$ must also be included; this is the item in the second column. The resulting belief set is characterised by the interpretations $\operatorname{Mod}(H) \cup\{a c, a\}=\{a b c, a b, a c, a\}$, which is the set of models of formula $a$, given in the third column. The result isn't a remainder set, since $C n^{h}(a \wedge(c \rightarrow b))$ is a logically stronger belief set that fails to imply $a \wedge b$. This last belief set, $C n^{h}(a \wedge(c \rightarrow b))$ appears in the second row of the table. It can be observed that the models of this belief set is made up of the models of $H$ together with the countermodel $a$, that is, the "induced model" in the first row.

As previously noted, there are three remainder sets, as indicated in the last column. As discussed, this result is problematic for the approaches of both Delgrande (2008) and Booth, Meyer, and Varzinczak (2009). For example, in none of the approaches in these papers is it possible to obtain $H \dot{-}_{e}(a \wedge b) \equiv a$, nor is it possible to obtain $H \dot{-}_{e}(a \wedge b) \equiv$ $((a \rightarrow b) \wedge(b \rightarrow a))$. But these possibilities would be desirable as potential contractions.

The diagnosis of the problem is now presumably clear. In the example, and for the countermodel given by $a \bar{b} c$, it is not possible to have a set of interpretations $\mathcal{M}$ satisfying:

1. $\mathcal{M}$ is closed under intersections

2. $\mathcal{M}=\operatorname{Mod}(H) \cup a \bar{b} c$

The solution also seems clear: From a semantic point of view, one wants the characteristic models of maxichoice candidates for $\mathrm{H}_{-}{ }_{e} \phi$ to consist of the characteristic models of $H$ together with a single interpretation from $\operatorname{Mod}(\top) \backslash \operatorname{Mod}(\phi)$. The resulting theories, called weak remainder sets, would correspond to the theories given in the third column in Figure 1; we explore this notion in the next subsection.

To conclude we note that it has been shown that (maxichoice) contraction based on remainder sets alone suffers from a triviality result analogous to that in AGM contraction.

Theorem 3 (Makinson, 2009) Let $a \in \mathbf{P}$ be an atom, and let $H$ be a Horn belief set with $a \rightarrow \perp \in H$. Let $\dot{-}$ be a maxichoice Horn contraction function based on remainder sets. Then for every atom $b$, at least one of $b$ and $b \rightarrow \perp$ is in $H \dot{-}(a \rightarrow \perp)+a$.

Hence if $a$ is false according to $H$, then contracting by $a \rightarrow \perp$ and then expanding by $a$ yields a belief set in which every atom is believed to be true or believed to be false. This is clearly far too unrealistic to be useful. 


\subsection{Horn Clause Contraction and Weak Remainder Sets}

The previous section showed that basing Horn contractions solely on remainder sets (or infra remainder sets) is problematic. We then suggested that an adequate version of contraction should be based on weak remainder sets where for belief set $H$ and formula $\phi \in H$, there is a 1-1 correspondence between countermodels of $\phi$ and weak remainder sets. In this section we develop Horn contraction based on weak remainder sets. We first give two constructions for weak remainder sets, in terms of belief sets and in terms of sets of models, and show that the constructions are equivalent. We then characterise maxichoice Horn contraction in terms of weak remainder sets, showing via a representation result that the characterisations are equivalent. Following this we similarly characterise partial meet contraction.

Definition 4 Let $H$ be a Horn belief set, and let $\phi$ be a Horn formula.

$H \Downarrow_{e} \phi$ is the set of sets of formulas such that

1. If $\phi \in H$ then: $H^{\prime} \in H \Downarrow_{e} \phi$ iff $H^{\prime}=H \cap m$ for some $m \in|\top| \backslash|\phi|$.

2. Otherwise $H \Downarrow_{e} \phi=\{H\}$.

$H^{\prime} \in H \Downarrow_{e} \phi$ is a weak remainder set of $H$ and $\phi$.

Observation 4 If $H^{\prime} \in H \Downarrow_{e} \phi$, then $H^{\prime}$ is a belief set, i.e., $H^{\prime}=C n^{h}\left(H^{\prime}\right)$.

In the above definition, $m$ is a maximal consistent set of formulas, and so corresponds to the set of formulas true in some interpretation. In this case the underlying interpretation would belong to $\operatorname{Mod}(\top) \backslash \operatorname{Mod}(\phi)$, which is to say that the underlying interpretation would be a countermodel of $\phi$.

Example 2 For $\mathbf{P}=\{a, b, c\}$, let $H=C n^{h}(a \wedge b)$ and $\phi=a \wedge b$.

For $m_{1}=C n^{h}(a \wedge \neg b \wedge \neg c) \in|\top| \backslash|\phi|$, we have $H \cap m_{1}=C n^{h}(a \wedge(c \rightarrow b))$.

For $m_{2}=C n^{h}(a \wedge \neg b \wedge c) \in|\top| \backslash|\phi|$, we have $H \cap m_{2}=C n^{h}(a)$.

Note that $\left(H \cap m_{2}\right) \subset\left(H \cap m_{1}\right)$, and also that full propositional closure gives $C n(H \cap$ $\left.m_{2}\right)=C n(a \wedge(b \vee c))$.

The previous definition specifies weak remainder sets in terms of maximal consistent sets of formulas. The next definition is similar, but is expressed directly in terms of countermodels of a formula.

Definition 5 Let $H$ be a Horn belief set, and let $\phi$ be a Horn formula. Define $H \|_{e} \phi$ by:

1. If $\phi \in H$ then: $H \|_{e} \phi$ is the set of sets of formulas such that $H^{\prime} \in H \|_{e} \phi$ iff there is $M \notin \operatorname{Mod}(\phi)$ such that $\operatorname{Mod}\left(H^{\prime}\right)=C l_{\cap}(\operatorname{Mod}(H) \cup\{M\})$.

2. Otherwise $H \|_{e} \phi=\{H\}$.

In our running example, $H \|_{e} \phi$ is given by the closure of the formulas in column 3 in Figure 1.

Perhaps not surprisingly, these two characterisations prove to be equivalent: 
Theorem 4 For $H$ a Horn belief set and $\phi$ a Horn formula:

$H \Downarrow_{e} \phi=H \|_{e} \phi$.

We are now in a position to define a Horn contraction operator. We start by defining a selection function, basically as is done in the AGM approach. Given a selection function, it is straightforward to define a maxichoice contraction operator, and following this, a partial meet contraction operator.

Definition 6 Let $H$ be a Horn belief set. $\gamma$ is a selection function for $H$ if, for every $\phi \in \mathcal{L}_{H C}$,

1. If $H \Downarrow_{e} \phi \neq \emptyset$ then $\emptyset \neq \gamma\left(H \Downarrow_{e} \phi\right) \subseteq H \Downarrow_{e} \phi$.

2. If $H \Downarrow e \phi=\emptyset$ then $\gamma(H \Downarrow e \phi)=\{H\}$.

Definition 7 Let $\gamma$ be a selection function on $H$ such that $\gamma\left(H \Downarrow_{e} \phi\right)=\left\{H^{\prime}\right\}$ for some $H^{\prime} \in H \Downarrow_{e} \phi$.

The maxichoice Horn contraction based on weak remainders is given by:

$$
H \cdot{ }_{w} \phi=\gamma\left(H \Downarrow_{e} \phi\right)
$$

Hence the result of a maxichoice contraction is characterised by a single weak remainder set.

We obtain the following representation result, relating the construction to a postulate set characterising contraction:

Theorem 5 Let $H$ be a Horn belief set. Then $\dot{-}_{w}$ is an operator of maxichoice Horn contraction based on weak remainders iff ${ }_{-}{ }_{w}$ satisfies the following postulates.

$\left(H \dot{-}_{w} 1\right) H \dot{-}_{w} \phi$ is a Horn belief set.

(closure)

$\left(H \dot{-}_{w} 2\right)$ If not $\vdash \phi$, then $\phi \notin H \dot{-}_{w} \phi$.

$\left(H \dot{-}_{w} 3\right) H \dot{-}_{w} \phi \subseteq H$.

(inclusion)

$\left(H \dot{-}_{w} 4\right)$ If $\phi \notin H$, then $H \dot{-}_{w} \phi=H$.

(vacuity)

$\left(H \dot{-}_{w} 5\right)$ If $\vdash \phi$ then $H \dot{-}_{w} \phi=H$

(failure)

$\left(H \dot{-}_{w} 6\right)$ If $\phi \equiv \psi$, then $H \dot{-}_{w} \phi=H \dot{-}_{w} \psi$.

(extensionality)

$\left(H \dot{-}_{w} 7\right)$ If $H \neq H \dot{-}_{w} \phi$ then $\exists \beta \in \mathcal{L}_{H C}$ such that $\{\phi, \beta\} \vdash \perp, H \dot{-}_{w} \phi \subseteq C n^{h}(\beta)$ and $\forall H^{\prime}$ s.t $H \dot{-}_{w} \phi \subset H^{\prime} \subseteq H$ we have $H^{\prime} \nsubseteq C n^{h}(\beta)$.

(maximality)

The first four postulates and $\left(H \dot{-}{ }_{w} 6\right)$ have obvious counterparts in the AGM contraction postulates. Notably, we do not obtain the recovery postulate. The following provides a counterexample. 
Example 3 Let $H=\mathcal{C} n(p \rightarrow q)$ and $\phi=p \wedge r \rightarrow q$.

Then $H \dot{-}{ }_{w} \phi \nvdash p \rightarrow q$, since $p \rightarrow q \vdash p \wedge r \rightarrow q$.

Thus $H \dot{-}_{w} \phi$ can be at most $\mathcal{C} n(\{p \wedge r \wedge i \rightarrow q \mid i \in \mathbf{P} \backslash\{p, r\}\}$.)

But $H_{-}{ }_{w} \phi+\phi \subseteq \mathcal{C} n(\{p \wedge r \wedge i \rightarrow q \mid i \in \mathbf{P} \backslash\{p, r\})+\phi \forall \forall p \rightarrow q$

and hence $H \dot{-}_{w} \phi+\phi \forall p \rightarrow q$.

Postulate $\left(\mathrm{H} \dot{-}_{w} 5\right)$ is derivable using the AGM postulates, but relies on the recovery postulate $(K-5)$ for its proof. Since we lack the recovery postulate, it is required here as a postulate, covering a special case, in its own right.

Postulate $\left(H \dot{-}{ }_{w} 7\right)$ is more complicated than the others, but it expresses the basic defining characteristic for maxichoice revision: If contraction is nontrivial (viz. $H \neq H \dot{-}_{w} \phi$ ), then some countermodel of $\phi$ is a model of $H \dot{-}_{w} \phi$. This is expressed by $\beta$, in that $\beta$ and $\phi$ are mutually inconsistent, $\mathrm{H}_{-}^{-}{ }_{w} \phi$ is a subset of the closure of $\beta$, and $\mathrm{H}_{-}{ }_{w} \phi$ is a maximal set of formulas for which this holds. This in turn means that, even though the recovery postulate does not hold, nonetheless the trivial contraction, in which the entire belief set is discarded, is excluded as a legal contraction operator. It can be verified that in Example 1 (see also Figure 1) that both countermodels $a \bar{b} c$ and $a \bar{b} \bar{c}$ fulfill the conditions on $\beta$ in $\left(H \dot{-}{ }_{w} 7\right)$, which is to say, this postulate captures the notion of weak remainder set.

We turn next to partial meet Horn contraction. The definition for partial meet Horn contraction is analogous to that in AGM contraction, but based on weak remainder sets:

Definition 8 Let $\gamma$ be a selection function on $H$ such that $\gamma\left(H \Downarrow_{e} \phi\right) \subseteq\left(H_{\Downarrow_{e}} \phi\right)$.

The partial meet Horn contraction based on weak remainders is given by:

$$
H \dot{-}_{p m} \phi=\bigcap \gamma\left(H H_{e} \phi\right)
$$

A representation result involves a modification of the last postulate for maxichoice contraction:

Theorem 6 Let $H$ be a Horn belief set. Then $\dot{-}_{p m}$ is an operator of partial meet Horn contraction based on weak remainders iff $\dot{-}_{p m}$ satisfies the postulates $\left(H \dot{-}_{w} 1\right)-\left(H \dot{-}_{w} 6\right)$ and:

$\left(H \dot{-}_{p m} 7\right)$ If $\beta \in H \backslash\left(H \dot{-}_{p m} \phi\right)$, then there is some $H^{\prime}$ such that $H \dot{-}_{p m} \phi \subseteq H^{\prime}, \phi \notin C n^{h}\left(H^{\prime}\right)$ and $\phi \in C n^{h}\left(H^{\prime} \cup\{\beta\}\right)$

(weak relevance)

Example 4 For our running example, the partial meet given by the first and last weak remainder sets in Figure 1 is given by

$$
C n^{h}((b \rightarrow a) \wedge(c \rightarrow a))
$$

In terms of models, it is characterised by the models of $a \wedge b$, together with the two countermodels given by atoms ac and $\emptyset$, and closed under intersections. 


\section{Supplementary Postulates}

In this section we investigate how the different proposals for Horn contraction operations behave with respect to the supplementary postulates $(K \dot{-} 7)$ and $(K \dot{-} 8)$. Throughout this section, we assume all selection functions to be transitively relational.

First we consider the operation of Horn partial meet e-contraction (Delgrande, 2008). The following example shows that, considering $\downarrow$ e as defined by Delgrande (see also Definition 2), Horn partial meet e-contraction does not satisfy $(K \dot{-} 7)$ :

Example 5 Let $H=C n^{h}(\{a \rightarrow b, b \rightarrow c, a \rightarrow d, d \rightarrow c\})$.

We then have

$$
\begin{aligned}
& H \downarrow_{e}(a \rightarrow c)=\left\{H_{1}, H_{2}, H_{3}, H_{4}\right\} \\
& H \downarrow_{e}(b \rightarrow c)=\left\{H_{5}\right\}
\end{aligned}
$$

where:

$$
\begin{aligned}
& H_{1}=C n^{h}(\{a \rightarrow b, a \rightarrow d\}), \\
& H_{2}=C n^{h}(\{a \rightarrow b, a \wedge c \rightarrow d, d \rightarrow c\}), \\
& H_{3}=C n^{h}(\{b \rightarrow c, a \wedge c \rightarrow b, a \rightarrow d\}), \\
& H_{4}=C n^{h}(\{a \wedge c \rightarrow b, b \rightarrow c, a \wedge c \rightarrow d, d \rightarrow c, a \wedge d \rightarrow b, a \wedge b \rightarrow d\}), \text { and } \\
& H_{5}=C n^{h}(\{a \rightarrow b, a \rightarrow d, d \rightarrow c\})
\end{aligned}
$$

Note that the two first elements of $H \downarrow_{e}(a \rightarrow c)$ are subsets of the single element of $H \downarrow_{e}(b \rightarrow c)$ and hence, cannot belong to $H \downarrow_{e}(a \rightarrow c \wedge b \rightarrow c)$.

$$
H \downarrow_{e}(a \rightarrow c \wedge b \rightarrow c)=\left\{H_{3}, H_{4}, H_{5}\right\}
$$

If we take a selection function based on a transitive relation between remainder sets that gives priority in the order in which they appear in this example, i.e., $H_{5} \prec H_{4} \prec H_{3} \prec$ $H_{2} \prec H_{1}$, we have:

$$
\begin{aligned}
& H-(a \rightarrow c)=H_{1} \\
& H-(b \rightarrow c)=H_{5} \\
& H-(a \rightarrow c \wedge b \rightarrow c)=H_{3}
\end{aligned}
$$

And we see that

$$
H-(a \rightarrow c) \cap H-(b \rightarrow c)=H_{1} \nsubseteq H_{3}=H-(a \rightarrow c \wedge b \rightarrow c)
$$

The same example shows that the operation does not satisfy $(K \dot{-} 8)$ :

$$
a \rightarrow c \notin H-(a \rightarrow c \wedge b \rightarrow c) \text { but } H-(a \rightarrow c \wedge b \rightarrow c) \not H-(a \rightarrow c) .
$$

If there are no further restrictions on the selection function, the same example also shows that contraction based on infra-remainders does not satisfy the supplementary postulates. Note that each remainder set in the example is also an infra-remainder and that the selection function always selects a single element. It suffices to assign all the remaining infra-remainders lower priority.

Now we can show that the operation of partial meet based on weak remainders (PMWR) has a better behaviour with respect to the supplementary postulates: 
Theorem 7 Partial meet based on weak remainders and a transitive relational selection function satisfies $(K \dot{-} 7)$ and $(K \dot{-} 8)$.

More recently, Zhuang and Pagnucco (2010a) have addressed Horn contraction from the point of view of epistemic entrenchment. They compare AGM contraction via epistemic entrenchment in classical propositional logic with contraction in Horn logics. A postulate set is provided and shown to characterise entrenchment-based Horn contraction. The fact that AGM contraction allows disjunctions of formulas, which in general will not be Horn, is handled by considering Horn strengthenings in their postulate set, which is to say, logically weakest Horn formulas that subsume the disjunction. In contrast to earlier work, their postulate set includes equivalents to the supplemental postulates, and so goes beyond the set of basic postulates. In more detail, Zhuang and Pagnucco (2010a) have the following:

Definition 9 For a given clause $\phi$, the set of its Horn strengthenings $(\phi)_{H}$ is the set such that $\psi \in(\phi)_{H}$ if and only if $\psi$ is a Horn clause and there is no Horn clause $\psi^{\prime}$ such that $\psi \subset \psi^{\prime} \subseteq \phi$.

Of the ten postulates given by Zhuang and Pagnucco (2010a) to characterize epistemic entrenchment Horn contraction (EEHC), postulates $(H \dot{-} 1),(H \dot{-} 2),(H \dot{-} 4),(H \dot{-} 6),(H \dot{-} 7)$ and $(H \dot{-} 8)$ correspond exactly to the AGM postulates with the same numbers. $(H \dot{-} 1)$, $(H \dot{-} 2),(H \dot{-} 3),(H \dot{-} 4)$ and $(H \dot{-} 6)$ correspond to postulates $\left(H \dot{-}{ }_{w} 1\right)-\left(H \dot{-}{ }_{w} 6\right)$ characterizing partial meet contraction based on weak remainders just defined. The three new postulates are:

$(H \dot{-} 5)$ If $\psi \in H \dot{-} \phi \wedge \psi$ then $\psi \in H \dot{-} \phi \wedge \psi \wedge \delta$

$(H \dot{-} 9)$ If $\psi \in H \backslash H \dot{-} \phi$ then $\forall \chi \in(\phi \vee \psi)_{H}, \chi \notin H \dot{-} \phi$

$(H \dot{-} 10)$ If $\forall \chi \in(\phi \vee \psi)_{H}, \chi \notin H \dot{-} \phi \wedge \psi$ then $\psi \notin H \backslash H \dot{-} \phi$

Subsequently, Zhuang and Pagnucco (2010b) have shown that transitively relational PMWR as defined above is more general than EEHC. This means that any operation satisfying their set of 10 postulates (which include $(K \dot{-} 7)$ and $(K \dot{-} 8))$ is a PMWR. We have seen that PMWR satisfies $(K \dot{-} 7)$ and $(K \dot{-} 8)$, hence, in order to compare PMWR and EEHC, we need to know whether PMWR satisfies $(H \dot{-} 5),(H \dot{-} 9)$ and $(H \dot{-} 10)$.

Theorem 8 PMWR satisfies $(H \dot{-} 5)$.

Zhuang (2012) has shown that weak relevance implies $(H \dot{-} 9)$, hence, PMWR satisfies $(H \dot{-} 9)$. PMWR in general does not satisfy $(H \dot{-} 10)$, as the following example shows:

Example 6 Let $H=C n^{h}(\{a, b\})$.

Then we have

$$
\begin{aligned}
& H \Downarrow_{e} a=\left\{H_{1}, H_{3}\right\} \text { and } \\
& H \Downarrow_{e}(a \wedge b)=\left\{H_{1}, H_{2}, H_{3}\right\},
\end{aligned}
$$

where 


$$
\begin{aligned}
& H_{1}=C n^{h}(\{b \rightarrow a, a \rightarrow b\}), \\
& H_{2}=C n^{h}(\{a\}) \text { and } \\
& H_{3}=C n^{h}(\{b\}) \text {. } \\
& \text { Assuming a selection function based on a transitive relation such that } H_{1} \prec H_{2} \text { and } \\
& \left.H_{1} \prec H_{3} \text { (and } H_{2} \preceq H_{3} \text { and } H_{3} \preceq H_{2}\right) \text {, we have } \\
& H-a=H_{3} \text { and } H-(a \wedge b)=H_{2} \cap H_{3} \\
& \text { Since }(a \vee b)_{H}=\{a, b\}, \text { we have that for any } \chi \in(a \vee b)_{H}, \chi \notin H-(a \wedge b), \text { but } b \in H-a .
\end{aligned}
$$

\section{Other Operators}

In this section we consider two contraction-like operators. The first, package contraction, is like contraction, but it is defined with respect to a set of formulas. The second operator, forget, can be regarded as a removal of an atom or set of atoms from the language of discourse.

\subsection{Package Contraction}

In AGM-style belief change in propositional logic, given a belief set $K$ and a set of formulas $\Phi$, the package contraction $K \dot{-}_{p} \Phi$ is a form of contraction in which no (non-tautological) member of $\Phi$ is in $K \dot{-}_{p} \Phi$. In propositional logic the effect of package contraction may be nearly, but not quite, obtained by contracting by the disjunction of elements in $\Phi$. To see the difference, consider where $\Phi=\{\phi, \psi\}$. Clearly, $\phi \vee \psi \notin K \dot{-}(\phi \vee \psi)$ whereas it seems that a "simultaneous contraction" $K \dot{-}{ }_{p}\{\phi, \psi\}$ should allow for the possibility of $\phi \vee \psi$ being true in the outcome.

As Booth, Meyer, and Varzinczak (2009) note, package contraction is of interest in Horn clause theories, given the limited expressivity of such theories. That is, if $\phi, \psi$ are Horn formulas, $H \dot{-}(\phi \vee \psi)$ will be undefined whenever $\phi \vee \psi$ is non-Horn (which, of course, will be most of the time). On the other hand, expressing a contraction of both $\phi$ and $\psi$ by $H \dot{-}_{p}\{\phi, \psi\}$ seems to be perfectly fine.

Our development of Horn package contraction is analogous to that of maxichoice Horn contraction based on weak remainders. Essentially, for a package contraction $H_{-}^{\circ} \Phi$, we ensure that for each $\phi \in \Phi$ a countermodel of $\phi$ is among the models of $H_{-}^{\circ} \Phi$.

Definition 10 Let $H$ be a Horn belief set, and let $\Phi=\left\{\phi_{1}, \ldots, \phi_{n}\right\}$ be a finite ${ }^{7}$ set of Horn formulas.

$H \Downarrow_{p} \Phi$ is the set of sets of formulas such that $H^{\prime} \in H \Downarrow_{p} \Phi$ iff

For every $1 \leq i \leq n, \exists m_{i}$ such that:

if $\forall \phi_{i}$ and $\phi_{i} \in H$ then $m_{i} \in|\top| \backslash\left|\phi_{i}\right|$; otherwise $m_{i}=\mathcal{L}_{H C}$; and $H^{\prime}=H \cap \bigcap_{i=1}^{n} m_{i}$.

In the next definition, the notion of a selection function on $H$ (Definition 6) is extended in the obvious fashion to apply to a set of Horn formulas.

7. Since we assume that the underlying language is finite, any set of formulas will be equivalent to a finite set of formulas, under logical equivalence of formulas. 


\section{Horn Clause Contraction Functions}

Definition 11 Let $\gamma$ be a selection function on $H$ such that $\gamma\left(H \Downarrow_{p} \Phi\right)=\left\{H^{\prime}\right\}$ for some $H^{\prime} \in H \Downarrow_{p} \Phi$.

The (maxichoice) package Horn contraction based on weak remainders is given by:

$$
H \dot{-}_{p} \Phi=\gamma\left(H \Downarrow_{p} \Phi\right)
$$

if $\emptyset \neq \Phi \cap H \nsubseteq C n^{h}(\top)$; and $H$ otherwise.

The following result relates elements of $H \Downarrow_{p} \Phi$ to weak remainders.

Theorem 9 Let $H$ be a Horn belief set and let $\Phi=\left\{\phi_{1}, \ldots, \phi_{n}\right\}$ be a set of Horn formulas where for $1 \leq i \leq n$ we have $\forall \phi_{i}$.

Then $H^{\prime} \in H_{\Downarrow p} \Phi$ iff for $1 \leq i \leq n$ there are $H_{i} \in H \Downarrow_{e} \phi_{i}$ and $H^{\prime}=\bigcap_{i=1}^{n} H_{i}$.

It follows immediately from this that any maxichoice Horn contraction defines a package contraction, and vice versa.

Corollary 1 Let $\dot{-}_{p}$ be an operator of maxichoice Horn package contraction. Then

$$
H \dot{-} \phi=H \dot{-}_{p} \Phi \quad \text { for } \Phi=\{\phi\}
$$

is an operator of maxichoice Horn contraction based on weak remainders.

Corollary 2 Let - be an operator of maxichoice Horn contraction based on weak remainders. Then

$$
H \dot{-}{ }_{p} \Phi=\bigcap_{\phi \in \Phi} H \dot{-} \phi
$$

is an operator of maxichoice Horn package contraction.

Example 7 Consider the Horn belief set $H=C n^{h}(\{a, b\})$ over $\mathbf{P}=\{a, b, c\}$. We want to determine elements of

$$
H \Downarrow_{p} \Phi=C n^{h}(\{a, b\}) \Downarrow_{p}\{a, b\} .
$$

There are a total of 14 elements in $H \Downarrow_{p} \Phi$ and so 14 candidate package contractions. These candidates can be described as follows:

1. There are 4 countermodels of a, given by:

$$
A=\{b c, b, c, \emptyset\}
$$

Thus there are four weak remainders corresponding to these countermodels, and so four candidates for maxichoice Horn contraction by a.

2. Similarly there are 4 countermodels of $b$ :

$$
B=\{a c, a, c, \emptyset\}
$$


3. Members of $H \Downarrow_{p} \Phi$ are given by

$$
C l_{\cap}(\operatorname{Mod}(H) \cup\{x\} \cup\{y\})
$$

for $x \in A$ and $y \in B$.

For example, for $x=b c, y=\emptyset$, we have that $C l_{\cap}(\operatorname{Mod}(H) \cup\{x\} \cup\{y\})=\{a b c, a b, b c, b, \emptyset\}$, which is the set of models of $(c \rightarrow b) \wedge(a \rightarrow b)$.

For $x=b c, y=a c$, we have that $C l_{\cap}(\operatorname{Mod}(H) \cup\{x\} \cup\{y\})=C n^{h}(\top)$; this holds for no other choice of $x$ and $y$.

What this example indicates informally is that there is a great deal of choice with respect to candidates for package contraction. To some extent, such a combinatorial explosion of possibilities is to be expected, given the fact that a formula will in general have a large number of countermodels, and that this is compounded by the fact that each formula in a package contraction will be associated with its own countermodel. However, it can also be noted that some candidate package contractions contain redundancies, in that a selected countermodel of $a$ may also be a countermodel of $b$, in which case there seems to be no reason to allow the possible incorporation of a separate countermodel of $b$. Consequently, we also consider versions of package contraction that in some sense yield a maximal belief set. However, first we provide results regarding package contraction.

We have the following result:

Theorem 10 Let $H$ be a Horn belief set. Then $\dot{-}_{p}$ is an operator of maxichoice Horn package contraction based on weak remainders iff $\dot{-}_{p}$ satisfies the following postulates:

$\left(H \dot{-}_{p} 1\right) H \dot{-}_{p} \Phi$ is a belief set.

(closure)

$\left(H \dot{-}_{p} 2\right)$ For $\phi \in \Phi$, if not $\vdash \phi$, then $\phi \notin H \dot{-}_{p} \Phi$

(success)

$\left(H \dot{-}_{p} 3\right) H \dot{-}_{p} \Phi \subseteq H$

(inclusion)

$\left(H \dot{-}_{p} 4\right) H \dot{-}_{p} \Phi=H \dot{-}_{p}(H \cap \Phi)$

(vacuity)

$\left(H \dot{-}_{p} 5\right) H \dot{-}_{p} \Phi=H \dot{-}_{p}\left(\Phi \backslash C n^{h}(\top)\right)$

(failure)

$\left(H \dot{-}_{p} 5 b\right) H \dot{-}{ }_{p} \emptyset=H$

(triviality)

$\left(H \dot{-}{ }_{p} 6\right)$ If $\phi \equiv \psi$, then

$$
H \dot{-}_{p}(\Phi \cup\{\phi\})=H \dot{-}_{p}(\Phi \cup\{\psi\})
$$

(extensionality)

$\left(H \dot{-}_{p} 7\right)$ If $H \neq H \dot{-}_{p} \Phi$ then for

$$
\Phi^{\prime}=\left(\Phi \backslash C n^{h}(\top)\right) \cap H=\left\{\phi_{1}, \ldots, \phi_{n}\right\}
$$

there is $\Delta=\left\{\beta_{1}, \ldots, \beta_{n}\right\}$ where for $1 \leq i \leq n$,

$$
\begin{aligned}
& \left\{\phi_{i}, \beta_{i}\right\} \vdash \perp \text { and } H \dot{-}_{p} \Phi \subseteq C n^{h}\left(\beta_{i}\right) \text { and } \\
& \forall H^{\prime} \text { s.t } H \cdot{ }_{p} \Phi \subset H^{\prime} \subseteq H, \exists \beta \in \Delta \text { such that } H^{\prime} \nsubseteq C n^{h}(\beta)
\end{aligned}
$$

(maximality) 
With the exception of the last postulate, these postulates are clear and reasonable: As usual, the result of package contraction is a belief set $\left(\mathrm{H}_{-}{ }_{p} 1\right)$. Moreover, each non-tautology in a set $\Phi$ is not believed following contraction $\left(H \dot{-}_{p} 2\right)$, and no formulas are added $\left(\mathrm{H}_{p}{ }_{p} 3\right)$. Contracting a formula not originally in $H$ has no effect on the contraction $\left(H \dot{-}_{p} 4\right)$, as does attempting to contract a tautology $\left(\mathrm{H}_{-}{ }_{p}\right)$. An empty contraction unsurprisingly has no effect $\left(H \dot{-}_{p} 5 b\right)$. As in other knowledge-level accounts, contraction is independent of the syntactic expression of formulas to be contracted $\left(\mathrm{H} \dot{-}_{p} 6\right)$. The last postulate $\left(\mathrm{H} \dot{-}_{p} 7\right)$ corresponds to the maximality postulate for contraction based on weak remainders. If a package contraction $\mathrm{H}^{\circ}{ }_{p} \Phi$ is nontrivial then each of the nontautologies in $\Phi$ that appear in $H$ satisfy the same maximality condition as the formula for contraction does for regular Horn contraction based on weak remainder sets. That is, package contraction essentially extends contraction to a set of formulas. This result is to be expected, given Theorem 9 which related elements of $H \Downarrow_{p} \Phi$ to weak remainders.

As discussed, a characteristic of maxichoice package contraction is that there are a large number of members of $H \Downarrow_{p} \Phi$, some of which may be logically quite weak. However it proves to be the case that we can eliminate some candidates via pragmatic concerns. We have that a package contraction $H_{-}^{-}{ }_{p} \Phi$ is a belief set $H^{\prime} \in H \Downarrow_{p} \Phi$ such that, informally, models of $H^{\prime}$ contain a countermodel for each $\phi_{i} \in \Phi$ along with models of $H$. In general, some interpretations will be countermodels of more than one member of $\Phi$, and so pragmatically, one can select minimal sets of countermodels. Hence in the case that $\bigcap_{i}\left(\operatorname{Mod}(\top) \backslash \operatorname{Mod}\left(\phi_{i}\right)\right) \neq \emptyset$, a single countermodel, that is some $m \in \bigcap_{i}\left(\operatorname{Mod}(\top) \backslash \operatorname{Mod}\left(\phi_{i}\right)\right)$, would be sufficient to yield a package contraction.

Now, it may be that $\bigcap_{i}\left(\operatorname{Mod}(\top) \backslash \operatorname{Mod}\left(\phi_{i}\right)\right)$ is empty. A simple example illustrates this case:

Example 8 Let $H=C n^{h}(a \rightarrow b, b \rightarrow a)$ where $\mathbf{P}=\{a, b\}$. Then $H \dot{-}_{p}\{a \rightarrow b, b \rightarrow a\}=$ $C n^{h}(\top)$. That is, the sole countermodel of $a \rightarrow b$ is $\{a\}$ while that of $b \rightarrow a$ is $\{b\}$. The intersection closure of these interpretations with those of $H$ is $\{a b, a, b, \emptyset\}=\operatorname{Mod}(\top)$.

Informally one can get around this by simply selecting a minimal set of models such that a countermodel of each member of $\Phi$ is in the set. These considerations yield the following definition:

Definition 12 Let $H$ be a Horn belief set, and let $\Phi=\left\{\phi_{1}, \ldots, \phi_{n}\right\}$ be a set of Horn formulas.

$H S(\Phi)$, the set of (minimal) hitting sets of interpretations with respect to $\Phi$, is defined by:

$S \in H S(\Phi)$ iff

1. $S \subseteq|\top|$

2. For every $1 \leq i \leq n$ where $\forall \phi_{i}$ and $\phi_{i} \in H, S \cap\left(|\top| \backslash\left|\phi_{i}\right|\right) \neq \emptyset$

3. For $S^{\prime} \subset S, S^{\prime} \cap\left(|\top| \backslash\left|\phi_{i}\right|\right)=\emptyset$ for some $1 \leq i \leq n$.

Thus we look for sets of sets of interpretations; elements of such a set $S$ are interpretations represented as maximal consistent sets of formulas (Condition 1). As well, this set $S$ 
contains a countermodel for each member of $\Phi$ (Condition 2) and moreover $S$ is a subsetminimal set that satisfies these conditions (Condition 3). Thus $S \in H S(\Phi)$ corresponds to a minimal set of countermodels of members of $\Phi$. As an aside, it can be noted that the notion of a hitting set is not new in general (Garey \& Johnson, 1979) nor in AI (Reiter, 1987).

Definition $13 H \Downarrow_{p h} \Phi$ is the set of sets of formulas such that $H^{\prime} \in H \Downarrow_{p h} \Phi$ iff $H^{\prime}=H \cap \bigcap_{m \in S}$ for some $S \in H S(\Phi)$.

Definition 14 Let $\gamma$ be a selection function on $H$ such that $\gamma\left(H \Downarrow_{p h} \Phi\right)=\left\{H^{\prime}\right\}$ for some $H^{\prime} \in H \Downarrow_{p h} \Phi$.

Define:

$$
H \dot{-}_{p h} \Phi=\gamma\left(H \Downarrow_{p h} \Phi\right)
$$

if $\emptyset \neq \Phi \cap H \nsubseteq C n^{h}(\top)$; and $H$ otherwise.

The following result follows straightforwardly.

Theorem $11 \mathrm{H}_{-}{ }_{p h} \Phi$ is an operator of maxichoice Horn package contraction.

Example 9 Consider the case where $H=C n^{h}(a, b), \mathbf{P}=\{a, b, c\}$.

1. Let $\Phi=\{a, b\}$.

It can be verified that the hitting sets are given by:

$$
\{\{a c, b c\},\{a, b c\},\{a c, b\},\{a, b\},\{c\},\{\}\}
$$

The corresponding elements of $H_{w_{p h}} \Phi$ are given by:

$$
\begin{aligned}
H \Downarrow p h \Phi=\{ & C n^{h}(\top), \\
& C n^{h}(c \rightarrow a), \\
& C n^{h}(c \rightarrow b), \\
& C n^{h}(c \rightarrow a, c \rightarrow b), \\
& C n^{h}(a \rightarrow b, b \rightarrow a), \\
& \left.C n^{h}(a \rightarrow b, b \rightarrow a, c \rightarrow a, c \rightarrow b)\right\} .
\end{aligned}
$$

Compare this with Example 7, where we have 14 candidate package contractions.

2. Let $\Phi=\{a, a \wedge b\}$. We obtain that

$$
\begin{aligned}
H \Downarrow_{p h} \Phi=\{ & C n^{h}(b), \\
& C n^{h}(b \wedge(c \rightarrow a)), \\
& C n^{h}(a \rightarrow b, b \rightarrow a), \\
& \left.C n^{h}(a \rightarrow b, b \rightarrow a, c \rightarrow a, c \rightarrow b)\right\} .
\end{aligned}
$$


Any set of formulas that satisfies Definition 13 clearly also satisfies Definition 11 . One can further restrict the set of candidate package contractions by replacing $S^{\prime} \subset S$ by $\left|S^{\prime}\right|<$ $|S|$ in the third part of Definition 12. In this case, the package contraction in Example 9, Part 1 would yield just the two candidates $C n^{h}(a \rightarrow b, b \rightarrow a)$ and $C n^{h}(a \rightarrow b, b \rightarrow a, c \rightarrow$ $a, c \rightarrow b)$. As well, of course, one could continue in the obvious fashion to define a notion of partial meet Horn package contraction. Given the limited use of such an operator, we omit the details.

\subsection{Forgetting in Horn Formulas}

This section examines another means of removing beliefs from an agent's belief set, that of forgetting (Lin \& Reiter, 1994; Lang \& Marquis, 2002). Forgetting is an operation on belief sets and atoms of the language; the result of forgetting an atom can be regarded as decreasing the language by that atom.

In addressing forgetting, it will be easier to work with a set of Horn clauses, rather than Horn formulas. Since there is no confusion, we will freely switch between sets of Horn clauses and the corresponding Horn formula comprising the conjunction of clauses in the set. Thus any time that a set appears as an element in a formula, it can be understood as standing for the conjunction of members of the set. Thus for sets of clauses $S_{1}$ and $S_{2}, S_{1} \vee S_{2}$ will stand for the formula $\left(\bigwedge_{\phi \in S_{1}} \phi\right) \vee\left(\bigwedge_{\phi \in S_{2}} \phi\right)$. Of course, all such sets are guaranteed to be finitely representable, since our language is finite.

We introduce the following notation for this section, where $S$ is a set of Horn clauses, and $T$ is now taken as a distinguished atom true in all interpretations.

- For $t \in\{\perp, \top\}, S[p / t]$ is the result of uniformly substituting $t$ for atom $p$ in every $\phi \in S$.

- $S_{\downarrow p}=\{\phi \in S \mid p$ does not occur in $\phi\}$

Assume without loss of generality that for Horn clause $\phi \in S$, that head $(\phi) \notin \operatorname{body}(\phi)$.

The following definition adapts the standard definition, attributed to George Boole, to forgetting in Horn clauses.

Definition 15 For set of Horn clauses $S$ and atom $p$, define forget $(S, p)$ to be $S[p / \perp] \vee$ $S[p / \top]$.

This is not immediately useful for us, since a disjunction is generally not Horn. However, the next result shows that this definition nonetheless leads to a Horn-definable forget operator. Recall that for clauses $c_{1}$ and $c_{2}$, expressed as sets of literals where $p \in c_{1}$ and $\neg p \in c_{2}$, that the resolvent of $c_{1}$ and $c_{2}$ is the clause $\left(c_{1} \backslash\{p\}\right) \cup\left(c_{2} \backslash\{\neg p\}\right)$. As well, recall that if $c_{1}$ and $c_{2}$ are Horn, then so is their resolvent.

In the following, $\operatorname{Res}(S, p)$ is the set of Horn clauses obtained from $S$ by carrying out all possible resolutions with respect to $p$.

Definition 16 Let $S$ be a set of Horn clauses and $p$ an atom. Define

$$
\begin{aligned}
& \operatorname{Res}(S, p)=\left\{\begin{array}{l}
\boldsymbol{\phi} \mid \exists \phi_{1}, \phi_{2} \in S \text { such that } \\
\\
\quad p \in \operatorname{body}\left(\phi_{1}\right) \text { and } p=\operatorname{head}\left(\phi_{2}\right), \text { and } \\
\left.\phi=\left(\operatorname{body}\left(\phi_{1}\right) \backslash\{p\} \cup \operatorname{body}\left(\phi_{2}\right)\right) \rightarrow \operatorname{head}\left(\phi_{1}\right)\right\}
\end{array}\right.
\end{aligned}
$$


Theorem $12 \operatorname{forget}(S, p) \equiv S_{\downarrow p} \cup \operatorname{Res}(S, p)$.

Corollary 3 Let $S$ be a set of Horn clauses and $p$ an atom. Then forget $(S, p)$ is equivalent to a set of Horn clauses.

Corollary 4 Let $S_{1}$ and $S_{2}$ be sets of Horn clauses and $p$ an atom. Then $S_{1} \equiv S_{2}$ implies that forget $\left(S_{1}, p\right) \equiv \operatorname{forget}\left(S_{2}, p\right)$.

There are several points of interest about these results. The theorem is expressed in terms of arbitrary sets of Horn clauses, and not just deductively-closed Horn belief sets. Hence the second corollary states a principle of irrelevance of syntax for the case for forgetting for belief bases. As well, the expression $S_{\downarrow p} \cup \operatorname{Res}(S, p)$ is readily computable, and so the theorem in fact provides a means of computing forget. Further, the approach clearly iterates for more than one atom. We obtain the additional result: ${ }^{8}$

\section{Corollary 5}

$$
\text { forget }(\operatorname{forget}(S, p), q) \equiv \operatorname{forget}(\operatorname{forget}(S, q), p) \text {. }
$$

Given this, we can define for a set of atoms $A$ that $\operatorname{forget}(S, \emptyset)=S$ and that

$$
\operatorname{forget}(S, A)=\operatorname{forget}(\operatorname{forget}(S, a), A \backslash\{a\})
$$

where $a \in A$. On the other hand, forgetting an atom may result in a quadratic blowup of the knowledge base.

Finally, it might seem that the approach allows for the definition of a revision operator — and a procedure for computing a revision — by using something akin to the Levi Identity. Let $\mathcal{A}(\phi)$ be the set of atoms appearing in (formula or set of formulas) $\phi$. Then:

$$
\operatorname{FRevise}(S, \phi) \stackrel{\text { def }}{=} \operatorname{forget}(S, \mathcal{A}(\phi))+\phi \text {. }
$$

In fact, this does yield a revision operator, but an operator that in general is far too drastic to be useful. To see this, consider a taxonomic knowledge base which asserts that whales are fish, whale $\rightarrow$ fish. Of course, whales are mammals, but in using the above definition to repair the knowledge base, one would first forget all knowledge involving whales, for example, that whales have fins, breathe air, give live birth, and so on. Such an example doesn't prove that there are no reasonable revision operators definable via forget, but it does show that a naïve approach is problematic. Moreover, these problems are not particular to Horn formulas, but rather any revision operator defined in terms of forgetting with respect to any underlying logic would be similarly problematic.

\section{Comparison among Constructions for Horn Contraction}

This section provides a technical summary of the differences between the various contraction operations defined on Horn belief sets:

- Every e-remainder is a weak remainder, but the converse is not true.

8. In fact, this is an easy consequence of the definition of forget. 
This is clearly seen in Figure 1. For a Horn theory $H$ and formula $\phi$, the e-remainders are the maximal subsets of $H$ that do not imply $\phi$. The weak remainders are characterised by the models of $H$ together with a single countermodel of $\phi$, and then closed under intersection. In propositional logic these notions would coincide; here they do not. As well, this means that weak remainders and partial meet are distinct notions, the latter corresponding to intersections of weak remainders.

Similarly, we obtain the following:

- Every e-remainder is an infra-remainder, but the converse is not true.

This is clear from Definition 3, and illustrated in Example 1.

We also have:

- Not all infra-remainders are weak-remainders.

Looking again at Figure 1, we see that the set $C n^{h}(\{c \rightarrow a, c \rightarrow b, a \rightarrow b\})$ is an infraremainder but not a weak remainder. It can however be obtained as the intersection of two remainders.

Consider Example 3.2 presented by Booth, Meyer and Varzinczak (2009), where $H=$ $C n^{h}(\{p \rightarrow q, q \rightarrow r\})$ and one wants to contract by $p \rightarrow r$ : In this case, the weak remainders coincide with the remainders. The set $\{p \wedge q \rightarrow r, p \wedge r \rightarrow q\}$ is an infra-remainder and cannot be obtained as the intersection of weak-remainders. The authors claim that this set is a desirable result of the contraction, but do not give any strong motivation.

Last, we have:

- Not all weak remainders are infra-remainders.

Infra-remainders, by definition, must contain full-meet and be contained in some remainder. Weak remainders are contained in some remainder (or are a remainder) but do not always contain full meet, as can be seen in the table in Figure 1. Full-meet in that example would contain $\{c \rightarrow a, c \rightarrow b\}$ and there are two weak remainders $\left(C n^{h}(a)\right.$ and $\left.C n^{h}(b)\right)$ which do not contain both formulas.

The last two items show that weak remainders and infra-remainders are independent concepts and their relation should be studied in more detail. These various relations are illustrated in Figure 2.

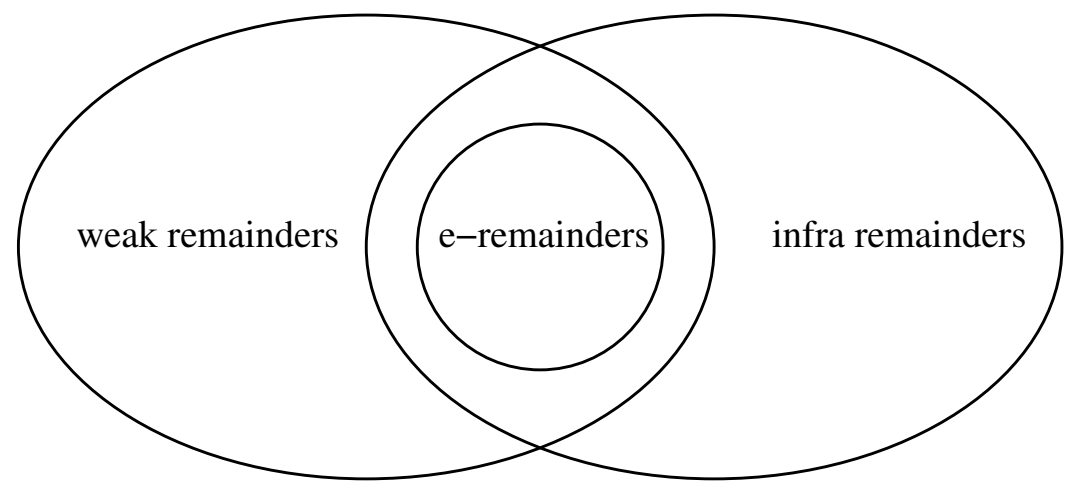

Figure 2 
The aforecited example of Booth, Meyer, and Varzinczak (2009) raises another point that deserves attention: For $H=C n^{h}(\{p \rightarrow q, q \rightarrow r\})$, we have $H \downarrow_{e}(p \rightarrow r)=H \Downarrow_{e}(p \rightarrow$ $r)=\left\{C n^{h}(\{p \rightarrow q\}), C n^{h}(\{q \rightarrow r, p \wedge r \rightarrow q\})\right\}$. There is an asymmetry here - while it is possible to obtain $C n^{h}(\{p \rightarrow q\})$ as the result of contraction, e-remainders, weak remainders or infra-remainders do not allow for $C n^{h}(\{q \rightarrow r\})$ as a possible outcome. This has motivated the study of Horn belief base contraction (Delgrande \& Wassermann, 2010), where one may obtain $C n^{h}(\{q \rightarrow r\})$, and where we think we may find other interesting alternatives.

Zhuang and Pagnucco have studied several forms of Horn Contraction, such as the Epistemic Entrenchment Horn Contraction (EEHC) mentioned earlier (2010a), Transitively Relational Partial-Meet Horn Contraction (TRPMHC) (2011) and Model-based Horn Contraction (MHC) (2012). These different operations are compared by Zhuang (2012). Partial meet based on weak remainders is more general than EEHC and MHC. However, when the selection function is required to be transitively relational, we obtain TRPMHC, which is equivalent to MHC.

\section{Conclusion}

In this paper we have explored belief contraction, and operators related to belief contraction, with respect to Horn theories. In the AGM approach there are two principal means of constructing contraction functions, via remainders or maximal subsets of a belief set that fail to imply a formula, and epistemic entrenchment, which incorporates a preference ordering on formulas. Here we focus on Horn contraction functions that can be defined by remainderlike constructions.

It proves to be the case that basing contraction directly on remainder sets, yielding what we call e-remainders, is problematic, in that the resulting approach is inexpressive and has undesirable properties. We also show that an alternative that has been proposed, of infra remainders suffers from the same problems. Based on an examination of model-theoretic considerations we developed an account of maxichoice Horn contraction in terms of weak remainder sets. The idea here is that the models of a contraction of a Horn belief set $H$ by a Horn formula $\phi$ are given by the models of $H$ together with a countermodel of $\phi$, closed under intersection (so as to yield a Horn theory). We then provided representation results for maxichoice Horn contraction as well as partial meet contraction, and compared them to other proposals in the literature.

We also study two other kinds of operators for giving up beliefs in Horn theories: package contraction and forgetting. The former involves contracting by a set of formulas, so that no formula in the set is believed, Again, we give a construction and postulate set, along with a corresponding representation result. The second operator, forgetting, can be thought of as a shrinking of the language of discourse.

This work is interesting since Horn clauses have found widespread use in areas such as logic programming, rule-based systems, deductive databases, and description logics. As well, since Horn reasoning is weaker than classical reasoning, this work sheds light on the foundations of belief change. A natural topic for future work is to consider Horn revision operators and study their relation to Horn contraction. A second topic for future work is to consider belief change in other logics which do not contain classical propositional logic. 


\section{Acknowledgments}

The first author was partially supported by a Canadian NSERC Discovery Grant. The second author was partially supported by the Brazilian National Research Council (CNPq), through grants 304043/2010-9 and 471666/2010-6. We thank Tommie Meyer, Marcio Ribeiro, David Makinson, and the anonymous reviewers for their helpful comments.

\section{Appendix A. Proofs of the Main Results}

Theorem 2: Let - be a Horn contraction function defined via a selection function as in (2) and based on (infra) remainder sets.

For $\phi \in H$ and $p$ inessential in $H$, we obtain that $(H \dot{-} \phi)+p \vdash \phi$.

Proof: Let $\phi=\phi_{1} \wedge \cdots \wedge \phi_{n}$ where each $\phi_{i}$ is a Horn clause. For Horn conjunct $\phi_{i}$ of $\phi$, we have $H \dot{-}_{e} \phi \mid=p \rightarrow \phi_{i}$. (To see this, note first that $\phi_{i}$ is a Horn clause, and so is of the form body $\rightarrow a$ for conjunction of atoms body and atom $a$. Since body $\rightarrow a \in H$ and $H$ is a Horn belief set, so also $p \wedge$ body $\rightarrow a \in H$. Since by assumption $p$ is not in body, it follows that $p \wedge \operatorname{body} \rightarrow a$ is in any remainder set of $H$ with respect to $\phi$. Then, $p \wedge$ body $\rightarrow a$ is logically equivalent to $p \rightarrow($ body $\rightarrow a)$, whence $H \dot{-}_{e} \phi \models p \rightarrow \phi_{i}$. $)$ Thus $\left(H \dot{-}_{e} \phi\right) \cup\{p\} \models \phi_{i}$ or $\left(H \dot{-}_{e} \phi\right)+p \models \phi_{i}$ for each conjunct of $\phi$, and so $\left(H \dot{-}_{e} \phi\right)+p \models \phi$.

Theorem 3: Let $a \in \mathbf{P}$ be an atom, and let $H$ be a Horn belief set with $a \rightarrow \perp \in H$. Let $\dot{-}$ be a maxichoice Horn contraction function based on remainder sets. Then for every atom $b$, at least one of $b$ and $b \rightarrow \perp$ is in $H \dot{-}(a \rightarrow \perp)+a$.

Proof: Suppose that for some atom $b$, neither of $b$ and $b \rightarrow \perp$ is in $H \dot{-}(a \rightarrow \perp)+a$, where $a \rightarrow \perp \in H$. Since $a$ is an atom, $a \rightarrow \perp$ is not a tautology, and as $a \rightarrow \perp \in H$, by construction, $H \dot{-}(a \rightarrow \perp)$ is an element of $H \downarrow_{e}(a \rightarrow \perp)$. This, together with the assumption $b, b \rightarrow \perp \notin H \dot{-}(a \rightarrow \perp)$, gives us (1) $(H \dot{-}(a \rightarrow \perp)) \cup\{b\} \vdash a \rightarrow \perp$ and (2) $(H \dot{-}(a \rightarrow \perp)) \cup\{b \rightarrow \perp\} \vdash a \rightarrow \perp$. (Results (1) and (2) are a consequence of the fact that since $H \dot{-}(a \rightarrow \perp)$ is a remainder set, it is a maximal set that fails to imply $a \rightarrow \perp$.) From (1) and (2) together, we have that $(H \dot{-}(a \rightarrow \perp)) \vdash a \rightarrow \perp$, contradicting the success postulate.

Lemma 1 Let $T$ be a set of propositional formulas. Then

$$
C l_{\cap}(\operatorname{Mod}(T))=\operatorname{Mod}(\operatorname{Horn}(C n(T))) .
$$

Proof: We have that $C l_{\cap}(\operatorname{Mod}(T))$ is the least set of models such that $\operatorname{Mod}(T) \subseteq$ $C l_{\cap}(\operatorname{Mod}(T))$ and where $C l_{\cap}(\operatorname{Mod}(T))=\operatorname{Mod}(H)$ for some Horn theory $H$. But this theory is just the least upper Horn approximation $T^{h}$ of $T$ (Selman \& Kautz, 1996), given by

$$
T^{h}=\{\alpha \mid T \vdash \alpha \text { where } \alpha \text { is a Horn prime implicate of } T\} .
$$

We have that $C n^{h}\left(T^{h}\right)=\operatorname{Horn}(C n(T))$ from which the result follows.

Theorem 4: For $H$ a Horn belief set and $\phi$ a Horn formula:

$$
H \Downarrow_{e} \phi=H \|_{e} \phi \text {. }
$$

\section{Proof:}


1. $H \Downarrow_{e} \phi \subseteq H \|_{e} \phi$ :

If $\phi \notin H$ or $\vdash \phi$ then $H \Downarrow_{e} \phi=H \|_{e} \phi=\{H\}$.

So assume that $\phi \in H$ and $\forall \phi$.

Let $H^{\prime} \in H \Downarrow_{e} \phi$; we show that $H^{\prime} \in H \|_{e} \phi$.

Since $H^{\prime} \in H \Downarrow_{e} \phi$, by definition $H^{\prime}=H \cap m$ for some $m \in|\top| \backslash|\phi|$, and so $\operatorname{Mod}\left(H^{\prime}\right)=$ $\operatorname{Mod}(H \cap m)$. $H$ and $m$ are Horn theories, thus $H \cap m$ is a Horn theory.

Using the fact that for Horn belief set $T, T=\operatorname{Horn}(C n(T))$, we have that $H \cap m=$ $\operatorname{Horn}(C n(H \cap m))$ and so $\operatorname{Mod}\left(H^{\prime}\right)=\operatorname{Mod}(\operatorname{Horn}(C n(H \cap m))$.

Applying Lemma 1 to $H \cap m$ we obtain that $\operatorname{Mod}\left(\operatorname{Horn}(C n(H \cap m))=C l_{\cap}(\operatorname{Mod}(C n(H \cap\right.$ $m)))$. Now, $C l_{\cap}(\operatorname{Mod}(C n(H \cap m)))=C l_{\cap}(\operatorname{Mod}(H \cap m))=C l_{\cap}((\operatorname{Mod}(H) \cup \operatorname{Mod}(m)))$.

By definition of $m$ as a maximal consistent Horn theory, there is $M \in \operatorname{Mod}(\top)$ such that $\operatorname{Mod}(m)=\{M\}$. Putting the above together we get that $\operatorname{Mod}\left(H^{\prime}\right)=$ $C l_{\cap}((\operatorname{Mod}(H) \cup M))$, that is, $H^{\prime} \in H \|_{e} \phi$.

2. $H \|_{e} \phi \subseteq H \Downarrow_{e} \phi:$

This part follows immediately by essentially taking the preceding part in reverse order.

Lemma 2 Maximality $\left(H \dot{-}_{w} 7\right)$ is equivalent to the following property, which we will call $\left(H \dot{-}_{w} 7^{\prime}\right)$ :

If $H \neq H \dot{-}_{w} \phi$ then $\exists m \in|\top| \backslash|\phi|$ s.t. $H \dot{-}^{\circ} \phi \subseteq m$ and $\forall H^{\prime}$ s.t. $H \dot{-}_{w} \phi \subset H^{\prime} \subseteq H$ we have $H^{\prime} \nsubseteq m$.

Proof: It is straightforward to show that the property implies $\left(H \dot{-}_{w} 7\right)$ : Let $\beta$ be the conjunction of literals appearing in $m$. Our language is finite, so $\beta$ is a well-defined formula. So $C n^{h}(\beta)=m$, and thus $\left(H \dot{-}_{w} 7\right)$ holds.

For the other direction, assume that $\left(H \dot{-}_{w} 7\right)$ holds.

Claim: For given $H$ and $\phi$, if $\beta$ satisfies the conditions in $\left(H \dot{-}{ }_{w} 7\right)$ then for any $p \in \mathbf{P}$, either $\beta \wedge p$ or $\beta \wedge(p \rightarrow \perp)$ also satisfies these conditions in $\left(\dot{H}_{-}{ }_{w} 7\right)$.

Proof of Claim: Clearly, if $\{\phi, \beta\}$ is inconsistent then so is $\{\phi, \beta \wedge l\}$ for $l \in$ $\{p, p \rightarrow \perp\}$; and if $H-\phi \subseteq C n^{h}(\beta)$ then $H^{\prime} \subseteq C n^{h}(\beta \wedge l)$ for $l \in\{p, p \rightarrow \perp\}$.

So we just need to show that for Horn theory $H^{\prime}$ where $H-\phi \subset H^{\prime} \subseteq H$, either $H^{\prime} \nsubseteq C n^{h}(\beta \wedge p)$ or $H^{\prime} \nsubseteq C n^{h}(\beta \wedge(p \rightarrow \perp))$.

Towards a contradiction, assume otherwise. Then $H^{\prime} \subseteq C n^{h}(\beta \wedge p)$ and $H^{\prime} \subseteq$ $C n^{h}(\beta \wedge(p \rightarrow \perp))$ and so $H^{\prime} \subseteq C n^{h}(\beta \wedge p) \cap C n^{h}(\beta \wedge(p \rightarrow \perp))$. But $C n^{h}(\beta)=$ $C n^{h}(\beta \wedge p) \cap C n^{h}(\beta \wedge p \rightarrow \perp)$, and consequently $H^{\prime} \subseteq C n^{h}(\beta)$. This contradicts that $\beta$ satisfies $\left(H \dot{-}^{-}{ }_{w}\right)$ for $H$ and $\phi$.

Hence our assumption was incorrect, and so $H^{\prime} \nsubseteq C n^{h}(\beta \wedge p)$ or $H^{\prime} \nsubseteq C n^{h}(\beta \wedge$ $(p \rightarrow \perp))$. 
We have just shown that if $\beta$ satisfies $\left(H \dot{-}{ }_{w} 7\right)$ for given $H$ and $\phi$, then so does one of $\beta \wedge p$ or $\beta \wedge(p \rightarrow \perp)$. An induction over (the finite set) $\mathbf{P}$ then establishes that if $\beta$ satisfies $\left(H-{ }_{w} 7\right)$ for given $H$ and $\phi$, then so does some $\beta^{\prime}$ where $\beta^{\prime} \vdash p$ or $\beta^{\prime} \vdash(p \rightarrow \perp)$ for every $p \in \mathbf{P}$. Hence $\beta^{\prime}$ is such that $C n^{h}\left(\beta^{\prime}\right) \in|T| \backslash|\phi|$, and thus taking $m=C n^{h}\left(\beta^{\prime}\right)$ satisfies the property.

Theorem 5: Let $H$ be a Horn belief set. Then $\dot{-}_{w}$ is an operator of maxichoice Horn contraction based on weak remainders iff $\dot{-}_{w}$ satisfies the following postulates.

$\left(H \dot{-}{ }_{w} 1\right) H \dot{-}{ }_{w} \phi$ is a Horn belief set.

$\left(H \dot{-}_{w} 2\right)$ If not $\vdash \phi$, then $\phi \notin H \dot{-}_{w} \phi$.

$\left(H \dot{-}_{w} 3\right) H \dot{-}_{w} \phi \subseteq H$.

(inclusion)

$\left(H \dot{-}_{w} 4\right)$ If $\phi \notin H$, then $H \dot{-}_{w} \phi=H$.

$\left(H \dot{-}_{w} 5\right)$ If $\vdash \phi$ then $H \dot{-}_{w} \phi=H$

$\left(H \dot{-}_{w} 6\right)$ If $\phi \equiv \psi$, then $H \dot{-}_{w} \phi=H \dot{-}_{w} \psi$.

(extensionality)

$\left(H \dot{-}_{w} 7\right)$ If $H \neq H \dot{-}_{w} \phi$ then $\exists \beta \in \mathcal{L}_{H C}$ such that $\{\phi, \beta\} \vdash \perp, H \dot{-}_{w} \phi \subseteq C n^{h}(\beta)$ and $\forall H^{\prime}$ s.t $H \dot{-}{ }_{w} \phi \subset H^{\prime} \subseteq H$ we have $H^{\prime} \nsubseteq C n^{h}(\beta)$.

(maximality)

\section{Proof:}

1. Construction to Postulates:

That the construction satisfies the first five postulates follows directly from the definitions of weak remainders and selection functions. To see that it satisfies $\left(H \dot{-}_{w} 6\right)$ we only have to note that $\phi \equiv \psi$ implies that $H \Downarrow_{e} \phi=H \Downarrow_{e} \psi$ and since $\gamma$ is a function, $H \dot{-}{ }_{w} \phi=H \dot{-}{ }_{w} \psi$.

To see that the construction satisfies $\left(H \dot{-}_{w} 7\right)$, suppose $H \neq H \dot{-}_{w} \phi$. This means that $H \Downarrow_{e} \phi \neq \emptyset$ and hence, there is $m \in|\top| \backslash|\phi|$ such that $H \dot{-}_{w} \phi=H \cap m$. Let $\beta$ be the conjunction of all literals appearing in $m$. Then, since $C n^{h}(\beta)=m$, we have that $\{\phi, \beta\}$ is inconsistent, $H \dot{-}_{w} \phi \subseteq C n^{h}(\beta)$ and $\forall H^{\prime}$ s.t $H \dot{-}_{w} \phi \subset H^{\prime} \subseteq H$ we have $H^{\prime} \nsubseteq C n^{h}(\beta)$.

2. Postulates to Construction:

The proof uses $\left(H \dot{-}_{w} 7^{\prime}\right)$ rather than $\left(H \dot{-}_{w} 7\right)$, as they were shown to be equivalent in Lemma 2.

Let $\dot{-}_{w}$ be an operator that satisfies $C n\left(H \dot{-}_{w} 1\right)-\left(H \dot{-}_{w} 7^{\prime}\right)$.

Let $\gamma$ be defined by $\gamma\left(H \Downarrow_{e} \phi\right)=\left\{H \dot{-}_{w} \phi\right\}$.

To show that $\gamma$ is a function: 
Assume that $H \Downarrow_{e} \phi=H \Downarrow_{e} \psi$; we need to show that $\gamma\left(H \Downarrow_{e} \phi\right)=\gamma\left(H \Downarrow_{e} \psi\right)$. If $\phi \notin H$, then $H \Downarrow_{e} \phi=\{H\}$ and since $H \Downarrow_{e} \phi=H \Downarrow_{e} \psi$, we have that $H \Downarrow_{e} \psi=H$, and hence $\psi \notin H$ or $\vdash \psi$. Then, by $\left(H \dot{-}_{w} 4\right)$ or $\left(H \dot{-}_{w} 5\right)$, $H \dot{-}_{w} \phi=H \dot{-}{ }_{w} \psi=H$ and by definition $\gamma\left(H \Downarrow_{e} \phi\right)=\gamma\left(H \Downarrow_{e} \psi\right)$.

Now let us consider the case where $\phi, \psi \in H$. Since $H \Downarrow_{e} \phi=H \Downarrow_{e} \psi$ we have that $\{H \cap m|m \in| \top|\backslash| \phi \mid\}=\{H \cap m|m \in| \top|\backslash| \psi \mid\}$.

It follows that $|\top| \backslash|\phi|=|\top| \backslash|\psi|$. To see this, suppose that $\{H \cap m \mid m \in$ $|\top| \backslash|\phi|\}=\{H \cap m|m \in| \top|\backslash| \psi \mid\}$ and $|\top| \backslash|\phi| \neq|\top| \backslash|\psi|$. Without loss of generality, suppose there is $m^{\prime} \in|T| \backslash|\phi|$ such that $m^{\prime} \notin|T| \backslash|\psi|$. Then $m^{\prime}$ is a maximal consistent theory that contains $\psi$. Since $\psi \in H$, we know that $\psi \in H \cap m^{\prime}$. This means that $H \cap m^{\prime} \in\{H \cap m|m \in| \top|\backslash| \phi \mid\}$, but $H \cap m^{\prime} \notin\{H \cap m|m \in| \top|\backslash| \psi \mid\}$, as for any $m \in|\top| \backslash|\psi|$ by definition $\psi \notin m$. This contradicts the initial hypothesis.

Since $|\top| \backslash|\phi|=|\top| \backslash|\psi|$ we get that $|\phi|=|\psi|$ and so $\phi \equiv \psi$. From $\left(H \dot{-}_{w} 6\right)$ we have $H \dot{-}_{w} \phi=H \dot{-}{ }_{w} \psi$, and so $\gamma\left(H \Downarrow_{e} \phi\right)=\gamma\left(H \Downarrow_{e} \psi\right)$.

If $\phi \notin H$, then from $\left(H \dot{-}_{w} 4\right)$ we have that $H \dot{-}_{w} \phi=H$. Similarly, if $\vdash \phi$, then from $\left(H \dot{-}_{w} 5\right)$ we again have that $H \dot{-}{ }_{w} \phi=H$.

Consequently assume that $\phi \in H$ and not $\vdash \phi$. We need to show that $H \dot{-}_{w} \phi \in H \Downarrow_{e} \phi$, that is, $H \dot{-}_{w} \phi=H \cap m$ for some $m \in|\top| \backslash|\phi|$.

Since not $\vdash \phi$, from $\left(H \dot{-}_{w} 2\right)$ we have $\phi \notin H \dot{-}_{w} \phi$; since $\phi \in H$ we then have that $H \neq H \dot{-}_{w} \phi$.

Since $H \neq H \dot{-}_{w} \phi$, from $\left(H \dot{-}{ }_{w} 7^{\prime}\right)$ we get that there is $m \in|\top| \backslash|\phi|$ such that $H \dot{-}_{w} \phi \subseteq$ $m$.

As well, $\left(H \dot{-}_{w} 3\right)$ gives $H \dot{-}_{w} \phi \subseteq H$, and so this with $H \dot{-}_{w} \phi \subseteq m$ implies that $H \dot{-}_{w} \phi \subseteq$ $(m \cap H)$.

We need to show that $H \dot{-}_{w} \phi=(m \cap H)$. Towards a contradiction assume that $H \dot{-}_{w} \phi \neq(m \cap H)$, that is to say, $H \dot{-}_{w} \phi \subset(m \cap H)$.

Let $\psi \in(m \cap H) \backslash\left(H \dot{-}_{w} \phi\right)$. Then

$$
H \dot{-}_{w} \phi \subset C n^{h}\left(H \dot{-}_{w} \phi \cup\{\psi\}\right) \subseteq m \cap H \subset H .
$$

But, substituting $C n^{h}\left(H \dot{-}{ }_{w} \phi \cup\{\psi\}\right)$ for $H^{\prime}$ in $\left(H \dot{-}_{w} 7^{\prime}\right)$ we get that $C n^{h}\left(H \dot{-}_{w} \phi \cup\right.$ $\{\psi\}) \nsubseteq m$, contradiction.

Hence the assumption that $H \dot{-}_{w} \phi \neq(m \cap H)$ is incorrect; hence $H \dot{-}_{w} \phi=(m \cap H)$ where $(m \cap H) \in H \Downarrow_{e} \phi$, which was to be shown.

Theorem 6: Let $H$ be a Horn belief set. Then $\dot{-}_{p m}$ is an operator of partial meet Horn contraction based on weak remainders iff $\dot{-}_{p m}$ satisfies the postulates $\left(H \dot{H}_{w} 1\right)-\left({ }^{\circ} \dot{-}_{w} 6\right)$ and:

$\left(H \dot{-}_{p m} 7\right)$ If $\beta \in H \backslash\left(H \dot{-}_{p m} \phi\right)$, then there is some $H^{\prime}$ such that $H \dot{-}_{p m} \phi \subseteq H^{\prime}, \phi \notin C n^{h}\left(H^{\prime}\right)$ and $\phi \in C n^{h}\left(H^{\prime} \cup\{\beta\}\right)$

(weak relevance) 


\section{Proof:}

1. Construction to Postulates:

$\left(H \dot{-}_{w} 1\right)$ follows from the fact that the intersection of Horn theories is a Horn theory. Postulates $\left(\mathrm{H}_{-}{ }_{w} 2\right)-\left(H \dot{-}{ }_{w} 6\right)$ follow immediately from the definitions of weak remainder, selection function and partial meet contraction.

To see that the construction satisfies weak relevance, note that if $\beta \in H \backslash H-\phi$, then there is some $X \in \gamma\left(H \Downarrow_{e} \phi\right)$ such that $\beta \notin X$. Since $\beta \in H$, then there is some $m \in|\top| \backslash|\phi|$ such that $\beta \notin m$ and $X=H \cap m$. Take $H^{\prime}=m$. Then $H-\phi \subseteq H^{\prime}$, $\phi \notin C n^{h}\left(H^{\prime}\right)$ and $\phi \in C n^{h}\left(H^{\prime} \cup\{\beta\}\right)=C n^{h}(\perp)$.

2. Postulates to Construction:

Let $\gamma\left(H \Downarrow_{e} \phi\right)=\left\{X \in H \Downarrow_{e} \phi \mid H \dot{-}_{p m} \phi \subseteq X\right\}$ if $H \Downarrow_{e} \phi \neq \emptyset$ and $\gamma\left(H \Downarrow_{e} \phi\right)=\{H\}$ otherwise. We have to show that: (1) $\gamma$ is a function; (2) $\gamma$ is a selection function; and $(3) \bigcap \gamma\left(H \Downarrow_{e} \phi\right)=H-\phi$.

If $\phi \notin H$, by $\left(H \dot{-}_{w} 4\right), H \dot{-}_{p m} \phi=H=\bigcap \gamma\left(H \Downarrow_{e} \phi\right)$. Assume then that $\phi \in H$.

(1) Let $H \Downarrow_{e} \phi_{1}=H \Downarrow_{e} \phi_{2}$. We must show that $\gamma\left(H \Downarrow_{e} \phi_{1}\right)=\gamma\left(H \Downarrow_{e} \phi_{2}\right)$. As in the proof for maxichoice contraction, $H \Downarrow_{e} \phi_{1}=H \Downarrow_{e} \phi_{2}$ implies that $\phi_{1} \equiv \phi_{2}$ and then, by Postulate $\left(H \dot{-}_{w} 6\right), H-\phi_{1}=H-\phi_{2}$. By the construction of $\gamma, \gamma\left(H \Downarrow_{e} \phi_{1}\right)=\gamma\left(H \Downarrow_{e} \phi_{2}\right)$.

(2) From the construction of $\gamma$, we know that $\gamma\left(H \Downarrow_{e} \phi\right) \subseteq H \Downarrow_{e} \phi$. So we have to show that if $H \Downarrow_{e} \phi \neq \emptyset$, then $\gamma\left(H \Downarrow_{e} \phi\right) \neq \emptyset$, and otherwise $\gamma\left(H_{\Downarrow e} \phi\right)=\{H\}$.

(i) If $H \Downarrow_{e} \phi \neq \emptyset$, then $H \neq \emptyset$ and $|\top| \backslash|\phi| \neq \emptyset$. By $\left(H \dot{-}_{w} 1\right)$ and $\left(H \dot{-}_{w} 2\right)$, $\phi \notin C n(H-\phi)$. Then there is $m \in|\top| \backslash|\phi|$ such that $H-\phi \subseteq m$. By $\left(H \dot{-}{ }_{w} 3\right)$, $H-\phi \subseteq H$, hence, $H-\phi \subseteq H \cap m \in \gamma(H \Downarrow \phi)$.

(ii) If $H \Downarrow_{e} \phi=\emptyset$, then $\vdash \phi$ and by $\left(H \dot{-}_{w} 5\right), H-\phi=H$.

(3) We know that $H-\phi \subseteq \bigcap \gamma\left(H \Downarrow_{e} \phi\right)$. Suppose there is $\beta \in \bigcap \gamma\left(H_{\Downarrow} \phi\right)$ such that $\beta \notin H-\phi$. Since $\bigcap \gamma\left(H \Downarrow_{e} \phi\right) \subseteq H, \beta \in H \backslash(H-\alpha)$ and by weak relevance we know that there is some $H^{\prime}$ such that $H-\phi \subseteq H^{\prime}, \phi \notin C n^{h}\left(H^{\prime}\right)$ and $\phi \in C n^{h}\left(H^{\prime} \cup\{\beta\}\right)$. Then there is $m \in|\mathrm{T}| \backslash|\phi|$ such that $H^{\prime} \subseteq m$ and $\beta \notin m$. Take $X=H \cap m$. Then $X \in H \Downarrow_{e} \phi$ and from $\left(H \dot{-}_{w} 3\right)$ we have that $H-\phi \subseteq X$ and hence, $X \in \gamma\left(H \Downarrow_{e} \phi\right)$. But $\beta \notin X$, which leads to a contradiction.

Theorem 7: Partial meet based on weak remainders and a transitive relational selection function satisfies $(K \dot{-} 7)$ and $(K \dot{-} 8)$.

\section{Proof:}

Let $\gamma$ be a selection function based on a transitive relation $\preceq$.

Since $|\top| \backslash|\alpha \wedge \beta|=(|\top| \backslash|\alpha|) \cup(|\top| \backslash|\beta|)$ and hence, $H \Downarrow_{e} \alpha \wedge \beta=H_{\Downarrow_{e}} \alpha \cup H_{\Downarrow_{e}} \beta$, in order to show that PMWR satisfies postulate (K-7), it suffices to show that

$$
\left({ }^{*}\right) \gamma\left(H \Downarrow_{e} \alpha \wedge \beta\right) \subseteq \gamma\left(H \Downarrow_{e} \alpha\right) \cup \gamma\left(H \Downarrow_{e} \beta\right) .^{9}
$$

9. This is called Choice-distributivity in the literature. 
Take $X \in \gamma\left(H \Downarrow_{e} \alpha \wedge \beta\right)$. We know that $X \in H \Downarrow_{e} \alpha$ or $X \in H \Downarrow_{e} \beta$. Suppose that $X \in H \Downarrow_{e} \alpha$, we have to show that $X \in \gamma\left(H \Downarrow_{e} \alpha\right)$. If $X \notin \gamma\left(H \Downarrow_{e} \alpha\right)$, then there is $X^{\prime} \in H \Downarrow_{e} \alpha$ such that $X \prec X^{\prime}$. But then $X^{\prime} \in H_{\Downarrow_{e}} \alpha \wedge \beta$ and $X \notin \gamma\left(H_{\Downarrow_{e}} \alpha \wedge \beta\right)$. The case where $X \in H \Downarrow_{e} \beta$ is analogous, thus $X \in \gamma\left(H \Downarrow_{e} \alpha\right)$ or $X \in \gamma\left(H \Downarrow_{e} \beta\right)$, which proves $\left(^{*}\right)$.

In order to show that PMWR satisfies postulate (K-8), let $\alpha \notin H-\alpha \wedge \beta$. We have to show that

$$
(* *) \gamma\left(H \Downarrow_{e} \alpha\right) \subseteq \gamma\left(H \Downarrow_{e} \alpha \wedge \beta\right)
$$

From $\alpha \notin H-\alpha \wedge \beta$ we know that $\gamma\left(H \Downarrow_{e} \alpha \wedge \beta\right)$ contains at least one element of $H \Downarrow_{e} \alpha$. Since $H \Downarrow_{e} \alpha \subseteq H \Downarrow_{e} \alpha \wedge \beta$ and $\gamma$ is based on $\preceq$, we have that $\gamma\left(H \Downarrow_{e} \alpha\right) \subseteq \gamma\left(H \Downarrow_{e} \alpha \wedge \beta\right)$.

Theorem 8: PMWR satisfies $(H \dot{-} 5)$.

\section{Proof:}

To see that PMWR satisfies $(H \dot{-} 5)$, first we have to note that $H \Downarrow_{e} \varphi \wedge \psi \subseteq H \Downarrow_{e} \varphi \wedge \psi \wedge \delta$. From $\psi \in H-\varphi \wedge \psi$, we know that $\psi \in X$ for every $X \in \gamma(H \Downarrow e \varphi \wedge \psi)$. We have to show that $\psi \in X$ for every $X \in \gamma\left(H_{\Downarrow} \varphi \wedge \psi \wedge \delta\right)$. Let $X \in \gamma\left(H \Downarrow_{e} \varphi \wedge \psi \wedge \delta\right)$. If $X \notin H_{\Downarrow} \varphi \wedge \psi$, then $X=H \cap m$ for $m$ a maximal, consistent Horn theory that does not contain $\varphi \wedge \psi \wedge \delta$ but contains $\varphi \wedge \psi$. Hence, $\psi \in X$. Otherwise, i.e., if $X \in H \Downarrow_{e} \varphi \wedge \psi$, we have to show that $X \in \gamma\left(H \Downarrow_{e} \varphi \wedge \psi\right)$. Suppose that $X \notin \gamma\left(H \Downarrow_{e} \varphi \wedge \psi\right)$, then there is $X^{\prime} \in H \Downarrow_{e} \varphi \wedge \psi$ such that $X^{\prime}<X$. But then $X^{\prime} \in H \Downarrow_{e} \varphi \wedge \psi \wedge \delta$ and $X$ cannot be an element of $\gamma\left(H \Downarrow_{e} \varphi \wedge \psi \wedge \delta\right)$. Hence, for every $X \in \gamma\left(H \Downarrow_{e} \varphi \wedge \psi \wedge \delta\right)$, we know that $\psi \in X$ and therefore, $\psi \in H-\varphi \wedge \psi \wedge \delta$.

Theorem 9: Let $H$ be a Horn belief set and let $\Phi=\left\{\phi_{1}, \ldots, \phi_{n}\right\}$ be a set of Horn formulas where for $1 \leq i \leq n$ we have $\forall \phi_{i}$.

Then $H^{\prime} \in H_{\Downarrow_{p}} \Phi$ iff for $1 \leq i \leq n$ there are $H_{i} \in H \Downarrow_{e} \phi_{i}$ and $H^{\prime}=\bigcap_{i=1}^{n} H_{i}$.

Proof: Let $H$ be a Horn belief set and $\Phi=\left\{\phi_{1}, \ldots, \phi_{n}\right\} \subset \mathcal{L}_{H C}$.

$\Longrightarrow$ Let $H^{\prime} \in H \Downarrow_{p} \Phi$.

From Definition 10 we have that $\exists m_{1}, \ldots, m_{n}$ such that $H^{\prime}=\bigcap_{i=1}^{n}\left(H \cap m_{i}\right)$ where

1. if $\phi_{i} \in H$ and $\forall \phi_{i}$ then $m_{i} \in|\top| \backslash\left|\phi_{i}\right|$;

2. otherwise $m_{i}=\mathcal{L}_{H C}$.

For each $i, 1 \leq i \leq n$, as above,

1. if $\phi_{i} \in H$ and $\forall \phi_{i}$ then by Definition $4, H_{i}=H \cap m_{i}$ satisfies the conditions for $H_{i} \in H_{\Downarrow} \phi_{i}$;

2. otherwise we have $m_{i}=\mathcal{L}_{H C}$ and so $H_{i}=H \cap m_{i}=H \cap \mathcal{L}_{H C}=H$ satisfies the conditions for $H_{i} \in H \Downarrow_{e} \phi_{i}$, again by Definition 4 .

$\Longleftarrow$ Consider some $\phi_{i} \in \Phi$ and let $H_{i} \in H_{\Downarrow} \Downarrow_{i}$.

From Definition 4 we have that

1. if $\phi_{i} \in H$ and $\forall \phi_{i}$ then $H_{i}=H \cap m$ for some $m \in|\top| \backslash\left|\phi_{i}\right|$; 
2. if $\phi_{i} \notin H$ or $\vdash \phi_{i}$ then $H_{i}=H$ or equivalently $H_{i}=H \cap m_{i}$ where $m_{i}=\mathcal{L}_{H C}$.

Consequently for each $i, 1 \leq i \leq n$, as above, $H^{\prime}=\bigcap_{i=1}^{n} H_{i}$ satisfies the conditions for $H^{\prime} \in H \Downarrow_{p} \Phi$ in Definition 10.

Theorem 10: Let $H$ be a Horn belief set. Then $\dot{-}_{p}$ is an operator of maxichoice Horn package contraction based on weak remainders iff $\dot{-}_{p}$ satisfies the following postulates:

$\left(H \dot{-}_{p} 1\right) H \dot{-}_{p} \Phi$ is a belief set.

$\left(H \dot{-}_{p} 2\right)$ For $\phi \in \Phi$, if not $\vdash \phi$, then $\phi \notin H \dot{H}_{p} \Phi$

$\left(H \dot{-}_{p} 3\right) H \dot{-}_{p} \Phi \subseteq H$

(inclusion)

$\left(H \dot{-}_{p} 4\right) H \dot{-}_{p} \Phi=H \dot{-}_{p}(H \cap \Phi)$

$\left(H \dot{-}_{p} 5\right) H \dot{-}_{p} \Phi=H \dot{-}_{p}\left(\Phi \backslash C n^{h}(\top)\right)$

$\left(H \dot{-}_{p} 5 b\right) \quad H \dot{-}{ }_{p} \emptyset=H$

$\left(H \dot{-}_{p} 6\right)$ If $\phi \equiv \psi$, then

$$
H \dot{-}_{p}(\Phi \cup\{\phi\})=H \dot{-}_{p}(\Phi \cup\{\psi\})
$$

(extensionality)

$\left(H \dot{-}_{p} 7\right)$ If $H \neq H \dot{-}_{p} \Phi$ then for

$$
\Phi^{\prime}=\left(\Phi \backslash C n^{h}(\top)\right) \cap H=\left\{\phi_{1}, \ldots, \phi_{n}\right\}
$$

there is $\Delta=\left\{\beta_{1}, \ldots, \beta_{n}\right\}$ where for $1 \leq i \leq n$,

$$
\begin{aligned}
& \left\{\phi_{i}, \beta_{i}\right\} \vdash \perp \text { and } H \dot{-}_{p} \Phi \subseteq C n^{h}\left(\beta_{i}\right) \text { and } \\
& \forall H^{\prime} \text { s.t } H \cdot{ }_{p} \Phi \subset H^{\prime} \subseteq H, \exists \beta \in \Delta \text { such that } H^{\prime} \nsubseteq C n^{h}(\beta)
\end{aligned}
$$

(maximality)

\section{Proof:}

1. Construction to Postulates:

$\left(H \dot{-}_{p} 1\right)$ is obvious.

For $\left(H \dot{-}_{p} 2\right)$, if $\phi \in H$, then Definition 10 ensures that for any $H^{\prime} \in H \Downarrow_{p} \Phi$ that $H^{\prime} \forall \phi$ and so $\phi \notin H^{\prime}$.

For $\left(H \dot{-}_{p} 3\right)$ we have that $H^{\prime} \in H \Downarrow_{p} \Phi$ implies that $H^{\prime}$ is of the form $H \cap X$; consequently $H^{\prime} \subseteq H$.

$\left(H \dot{-}_{p} 4\right)$ and $\left(H \dot{-}_{p} 5\right)$ are a direct consequence of the special cases in Definition 10 for $\phi \in \Phi$ where $\phi \notin H$ or $\vdash \phi$ respectively.

$\left(H \dot{-}_{p} 5 b\right)$ is vacuously satisfied by Definition 10 , while for $\left(H \dot{-}_{p} 6\right)$, the form of any $H^{\prime} \in H \Downarrow_{p} \Phi$ is easily seen to be independent of the syntactic form of members of $\Phi$.

For $\left(H \dot{-}_{p} 7\right)$, let $X \in H \Downarrow_{p} \Phi$ where $\Phi=\left\{\phi_{1}, \ldots, \phi_{n}\right\}$. By appeal to $\left(H \dot{-}_{p} 4\right)$ and $\left(H-{ }_{p} 5\right)$ we can assume without loss of generality that $\phi \in \Phi$ implies that $\forall \phi$ and $\phi \in H$. Let $m_{1}, \ldots, m_{n}$ be as specified in Definition 10 . Then $m_{1}, \ldots, m_{n}$ satisfy 
the conditions on $\beta_{1}, \ldots, \beta_{n}$ in $\left(H \dot{-}_{p} 7\right)$ : Since $m_{i} \in|\top| \backslash\left|\phi_{i}\right|$, so $\left\{\phi_{i}, \beta_{i}\right\} \vdash \perp$. Since $X=H \cap \bigcap_{i=1}^{n} m_{i}$, so $X \subseteq C n^{h}\left(m_{i}\right)=m_{i}$. Last, we need to show that for any belief set $H^{\prime}$ where $X \subset H^{\prime} \subseteq H$, that for some $m_{i}$ in our list, $H^{\prime} \nsubseteq C n^{h}\left(m_{i}\right)=m_{i}$. But this is a direct consequence of the fact that $X=H \cap \bigcap_{i=1}^{n} m_{i}$.

2. Postulates to Construction:

Let $\dot{-}_{p}$ satisfy Postulates $\left(H \dot{-}_{p} 1\right)-\left(H \dot{-}_{p} 7\right)$, and let $H$ be a Horn belief set and $\Phi \subseteq$ $\mathcal{L}_{H C}$. Let $\Delta$ be as specified in $\left(H \dot{-}_{p} 7\right)$ and for $\phi \in \Phi$, define $H-\phi$ by:

(a) If $\vdash \phi$ or $\phi \notin H$ then $H-\phi=H$.

(b) Otherwise for the $\beta \in \Delta$ corresponding to $\phi, H-\phi$ is the maximum set of formulas such that $H \dot{-}_{p} \Phi \subseteq H-\phi \subset H$ and $H-\phi \subseteq C n^{h}(\beta)$.

Using Theorem 5, it is easily shown that - is an operator of maxichoice Horn contraction.

This implies that there is a selection function $\gamma$ such that $H-\phi=\gamma\left(H \Downarrow_{e} \phi\right)$ for every $\phi \in \Phi$.

Therefore, by Theorem 9 we have that $H \dot{-}_{p} \Phi=\bigcap_{\phi \in \Phi} H-\phi=H^{\prime}$ is such that $H^{\prime} \in H \Downarrow_{p} \Phi$.

Theorem 12: $\operatorname{forget}(S, p) \equiv S_{\downarrow p} \cup \operatorname{Res}(S, p)$.

Proof: Let $S$ be a finite set of nontautological Horn clauses. For $p \in \mathbf{P}$, define:

$$
\begin{aligned}
S_{h} & =\{c \in S \mid p=\operatorname{head}(c)\} \\
S_{b} & =\{c \in S \mid p \in \operatorname{body}(c)\}
\end{aligned}
$$

As well, we have already defined: $S_{\downarrow p}=\{c \in S \mid p$ does not occur in $c\}$.

We obtain:

$$
\begin{aligned}
\operatorname{forget}(S, p) \equiv & S[p / \perp] \vee S[p / \top] \\
\equiv & \left(S_{h}[p / \perp] \cup S_{b}[p / \perp] \cup S_{\downarrow p}[p / \perp]\right) \vee \\
& \quad\left(S_{h}[p / \top] \cup S_{b}[p / \top] \cup S_{\downarrow p}[p / \top]\right) \\
\equiv & \left(S_{h}[p / \perp] \cup\{\top\} \cup S_{\downarrow p}\right) \vee\left(\{\top\} \cup S_{b}[p / \top] \cup S_{\downarrow p}\right) \\
\equiv & \left(S_{h}[p / \perp] \cup S_{\downarrow p}\right) \vee\left(S_{b}[p / \top] \cup S_{\downarrow p}\right) \\
\equiv & S_{\downarrow p} \cup\left(S_{h}[p / \perp] \vee S_{b}[p / \top]\right) \\
\equiv & S_{\downarrow p} \cup\left\{c_{1} \cup c_{2} \mid c_{1} \in S_{h}[p / \perp] \text { and } c_{2} \in S_{b}[p / \top]\right\} \\
\equiv & S_{\downarrow p} \cup \operatorname{Res}(S, p) \square
\end{aligned}
$$

\section{References}

Alchourron, C. E., \& Makinson, D. (1985). On the logic of theory change: Safe contraction. Studia Logica, 44(4), 405-422. 
Alchourrón, C., Gärdenfors, P., \& Makinson, D. (1985). On the logic of theory change: Partial is meet contraction and revision functions. Journal of Symbolic Logic, 50(2), $510-530$.

Anderson, A., \& Belnap Jr., N. (1975). Entailment: The Logic of Relevance and Necessity, Vol. I. Princeton University Press.

Baader, F., Calvanese, D., McGuiness, D., Nardi, D., \& Patel-Schneider, P. (Eds.). (2007). The Description Logic Handbook (second edition). Cambridge University Press.

Booth, R., Meyer, T., Varzinczak, I., \& Wassermann, R. (2011). On the Link between Partial Meet, Kernel, and Infra Contraction and its Application to Horn Logic. Journal of Artificial Intelligence Research, 42, 31-53.

Booth, R., Meyer, T., \& Varzinczak, I. (2009). Next steps in propositional Horn contraction. In Proceedings of the International Joint Conference on Artificial Intelligence, pp. 702-707, Pasadena, CA.

Creignou, N., Papini, O., Pichler, R., \& Woltran, S. (2012). Belief revision within fragments of propositional logic. In Brewka, G., Eiter, T., \& McIlraith, S. A. (Eds.), Proceedings of the Thirteenth International Conference on the Principles of Knowledge Representation and Reasoning. AAAI Press.

Delgrande, J., \& Wassermann, R. (2010). Horn clause contraction functions: Belief set and belief base approaches. In Lin, F., \& Sattler, U. (Eds.), Proceedings of the Twelfth International Conference on the Principles of Knowledge Representation and Reasoning, pp. 143-152, Toronto. AAAI Press.

Delgrande, J. (2008). Horn clause belief change: Contraction functions. In Brewka, G., \& Lang, J. (Eds.), Proceedings of the Eleventh International Conference on the Principles of Knowledge Representation and Reasoning, pp. 156-165, Sydney, Australia. AAAI Press.

Delgrande, J., \& Peppas, P. (2011). Revising Horn Theories. In Twenty-Second International Joint Conference on Artificial Intelligence, pp. 839-844.

Delgrande, J., \& Wassermann, R. (2011). Topics in Horn contraction: Supplementary postulates, package contraction, and forgetting. In IJCAI-11 Workshop on Nonmonotonic Reasoning, Action and Change (NRAC-11), pp. 87-94, Barcelona, Spain.

Eiter, T., \& Gottlob, G. (1992). On the complexity of propositional knowledge base revision, updates, and counterfactuals. Artificial Intelligence, 57(2-3), 227-270.

Flouris, G., Plexousakis, D., \& Antoniou, G. (2004). Generalizing the AGM postulates: Preliminary results and applications. In Proceedings of the 10th International Workshop on Non-Monotonic Reasoning (NMR-04), pp. 171-179, Whistler BC, Canada.

Gärdenfors, P. (1988). Knowledge in Flux: Modelling the Dynamics of Epistemic States. The MIT Press, Cambridge, MA.

Gärdenfors, P., \& Makinson, D. (1988). Revisions of knowledge systems using epistemic entrenchment. In Proc. Second Theoretical Aspects of Reasoning About Knowledge Conference, pp. 83-95, Monterey, Ca. 
Garey, M., \& Johnson, D. (1979). Computers and Intractability: A Guide to the Theory of NP-Completeness. W.H. Freeman and Co., New York.

Grove, A. (1988). Two Modellings for Theory Change. Journal of Philosophical Logic, 17, $157-170$.

Hansson, S. O. (1999). A Textbook of Belief Dynamics. Applied Logic Series. Kluwer Academic Publishers.

Khardon, R. (1995). Translating between Horn representations and their characteristic models. Journal of Artificial Intelligence Research, 3, 349-372.

Lakemeyer, G., \& Levesque, H. (2000). The Logic of Knowledge Bases. MIT Press, Cambridge, MA.

Lang, J., \& Marquis, P. (2002). Resolving inconsistencies by variable forgetting. In Proceedings of the Eighth International Conference on the Principles of Knowledge Representation and Reasoning, pp. 239-250, San Francisco. Morgan Kaufmann.

Langlois, M., Sloan, R., Szörényi, B., \& Turán, G. (2008). Horn complements: Towards Horn-to-Horn belief revision. In Proceedings of the AAAI National Conference on Artificial Intelligence, Chicago, Il.

Liberatore, P. (2000). Compilability and compact representations of revision of Horn knowledge bases. ACM Transactions on Computational Logic, 1(1), 131-161.

Lin, F., \& Reiter, R. (1994). Forget it!. In AAAI Fall Symposium on Relevance, New Orleans.

Makinson, D. (2009) Personal communication.

Peppas, P. (2008). Belief revision. In van Harmelen, F., Lifschitz, V., \& Porter, B. (Eds.), Handbook of Knowledge Representation, pp. 317-359. Elsevier Science, San Diego, USA.

Reiter, R. (1987). A theory of diagnosis from first principles. Artificial Intelligence, 32(1), $57-96$.

Rott, H. (1992). On the logic of theory change: More maps between different kinds of contraction functions. In Gärdenfors, P. (Ed.), Belief Revision, No. 29 in Cambridge Tracts in Theoretical Computer Science, pp. 122-141. Cambridge University Press.

Selman, B., \& Kautz, H. (1996). Knowledge compilation and theory approximation. Journal of the ACM, 43(2), 193-224.

Zhuang, Z., \& Pagnucco, M. (2010a). Horn contraction via epistemic entrenchment. In Janhunen, T., \& Niemelä, I. (Eds.), Logics in Artificial Intelligence - 12th European Conference (JELIA 2010), Vol. 6341 of Lecture Notes in Artificial Intelligence, pp. 339-351. Springer Verlag.

Zhuang, Z., \& Pagnucco, M. (2010b). Two methods for constructing Horn contractions. In Li, J. (Ed.), AI 2010: Advances in Artificial Intelligence - 23rd Australasian Joint Conference, Vol. 6464 of Lecture Notes in Artificial Intelligence, pp. 72-81. Springer Verlag. 
Zhuang, Z. (2012). Belief Change Under the Horn Fragment of Propositional Logic. Ph.D. thesis, School of Computer Science and Engineering - University of New South Wales.

Zhuang, Z., \& Pagnucco, M. (2011). Transitively relational partial meet Horn contraction. In Proceedings of the Twenty-Second International Joint Conference on Artificial Intelligence, pp. 1132-1138, Barcelona, Spain.

Zhuang, Z., \& Pagnucco, M. (2012). Model based Horn contraction. In Proceedings of the Thirteenth International Conference on the Principles of Knowledge Representation and Reasoning, Rome, Italy. 